\title{
WEAK CONVERGENCE OF PROBABILITY MEASURES ON METRIC SPACES OF NONLINEAR OPERATORS
}

\author{
WEN HSIANG WEI
}

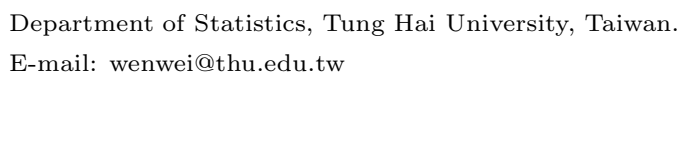

Abstract

The conditions for weak convergence of a sequence of probability measures on metric spaces of nonlinear operators defined on some subsets of a real separable Banach space are established. The nonlinear operators of interest include either continuous operators or cadlag (continu à droite, limites à gauche) operators defined in this article. As the domains of the operators are certain compact sets, the limiting probability measures are the generalizations of the Wiener measure and the Poisson measure on the metric spaces of continuous and cadlag real functions defined on the unit interval, respectively. As the limiting probability measure is the generalized Wiener measure, the result is a generalization of Donsker's theorem.

\section{Introduction}

Let $C([0,1], R)$ and $D([0,1], R)$ be the complete metric spaces of continuous real functions endowed with the uniform topology (see [4], Chapter 2, p. 80) and cadlag real functions endowed with the Skorohod topology (see [4], Chapter 3, p. 125) on the unit interval, respectively. Let $\mathcal{C}$ and $\mathcal{D}$ be the corresponding Borel $\sigma$-fields. Weak convergence of a sequence of probability measures on either $\{C([0,1], R), \mathcal{C}\}$ or $\{D([0,1], R), \mathcal{D}\}$ given some sufficient conditions has been proved (see [4], Theorem 7.1, Theorem 7.5, and Theorem 13.1). Basically, the tightness of the sequence of probability measures and weak convergence of the finite-dimensional distributions are two main conditions. As the limiting measure is the Wiener measure, the result is

Received September 1, 2015.

AMS Subject Classification: 60F17, 60F05.

Key words and phrases: Banach spaces, Donsker's theorem, Poisson measure, Polish spaces, weak convergence, Wiener measure. 
Donsker's theorem (see [4], Theorem 8.2). Further, the limiting probability measure can be the Poisson measure (see [4], Example 12.3). In addition, weak convergence theory in $D([0,1], R)$ can be applied to prove the convergence of the empirical process to the Brownian bridge (see [4], Theorem 14.3; also see [5], Chapter 1.1).

Relative little has been done for weak convergence of probability measures on the space of continuous operators, possibly nonlinear, from a subset of a real separable Banach space $X$ to a real separable Banach space $Y$ (see [2], p. 30; 16], Section 1.5) or to the space of certain operators from the subset of the space $X$ to the space $Y$, i.e., the results for operator-valued random variables or probability measures on the space of nonlinear operators. Operator theory has been at the heart of research in analysis (see [1]). Moreover, as implied by [13], considering nonlinear case should be essential. The following are some examples related to nonlinear operators and the results developed in this article. Let $X$ and $Y$ be normed spaces in these examples.

Example 1. The continuous operators from $X$ into $Y$.

Example 2. Suppose that the underlying $d$ th order partial differential equation can be expressed as

$$
\begin{aligned}
& D\left[\mu, x(\mu), \frac{\partial x(\mu)}{\partial \mu_{1}}, \ldots, \frac{\partial^{|\alpha|} x(\mu)}{\partial \mu_{j_{1}}^{\alpha_{1}} \cdots \partial \mu_{j_{r}}^{\alpha_{r}}}, \cdots, \frac{\partial^{d} x(\mu)}{\partial \mu_{p}^{d}}\right] \\
& =F(x)(\mu) \\
& =0
\end{aligned}
$$

where $\mu=\left(\mu_{1}, \ldots, \mu_{p}\right)^{t} \in R^{p}, p>1, D$ is a real or complex function, $F: \operatorname{Dom}(F) \subset X \rightarrow Y$ is an operator, $\alpha=\left(\alpha_{1}, \ldots, \alpha_{r}\right),|\alpha|=\sum_{k=1}^{r} \alpha_{k} \leq d$, $\alpha_{k}$ are non-negative integers, $\left\{j_{1}, \ldots, j_{r}\right\} \subset\{1, \ldots, p\}$, and where $\operatorname{Dom}(F)$ is the domain of the operator $F$. As indicated by [8], the great advantage of interpreting PDE problem in the operator form is that the general and elegant results of functional analysis can be used to study the solvability of various equations involving the differential operator. For nonlinear differential equation, the operator might not be linear. As the underlying equation is not deterministic and is involving some random quantities, the resulting differential operator is the realization of an operator-valued random variable. 
Example 3. Consider the mathematical programming problem of which goal is to find the minimizer

$$
\hat{F}=\underset{F \in S}{\arg \min } T(F)
$$

subject to

$$
\begin{aligned}
g_{i}(F) & \leq 0, i=1, \ldots, I, \\
h_{j}(F) & =0, j=1, \ldots, J,
\end{aligned}
$$

where $S$ is a subset consisting of nonlinear operators from $X$ into $Y$ and $T$, $g_{i}$ and $h_{j}$ are functionals defined on $S$.

Example 4. One of the fundamental quantities in robustness theory is the Huber function. One of the generalizations of the Huber function to the infinite dimensional spaces is $F: X \rightarrow X$ (see [14]),

$$
F(x)=x \min \left[1, \frac{\|c\|_{Z_{1}}}{\|U(x)\|_{Z_{2}}}\right],
$$

if $\|U(x)\|_{Z_{2}} \neq 0$ and $F(x)=x$ if $\|U(x)\|_{Z_{2}}=0$, where $Z_{1}$ and $Z_{2}$ are normed spaces, $c \in Z_{1}$ is a bounded function, $U: X \rightarrow Z_{2}$ is considered as a continuous linear operator. Note that $F$ is not a linear operator.

Example 5. Consider the following nonparametric regression model in statistics,

$$
y=F(x)+\varepsilon,
$$

where $y$ is a $Y$-valued random variables, $F$ defined on some subset of $X$ is a $Y$-valued nonlinear operator, and $\varepsilon$ is a $Y$-valued random variable. Relatively few theoretical results such as consistency and weak convergence of the nonlinear estimators (operators) have been established. However, one theorem concerning weak convergence of the operators of interest (see [15]) is based on the result developed in this article .

Developing useful results for the operators in the above examples holds promise for the wide applications of nonlinear functional analysis to a variety of scientific areas. As considering the measure space of the nonlinear operators, the occurring problem is when can a sequence of measurable functions on the space converge in different notions? Weak convergence of a sequence 
of measures is one mode of convergence and is one of main research topics in mathematics, particularly in probability or statistics. Note that weak convergence theory corresponding to nonlinear operators might also hold the promise for wide applications to the above examples.

The goal of this article is to establish the conditions for weak convergence of a sequence of probability measures on the metric space of nonlinear operators from the possibly unbounded subset of $X$ to the space $Y$. The proofs for these results mainly follow the ones of [4]. In next section, the results concerning weak convergence of a sequence of probability measures on the complete and separable metric space of continuous operators defined on the compact set of $X$ are given. The sufficient conditions corresponding to the tightness of the sequence of probability measures and weak convergence of the finite-dimensional distributions are established. Furthermore, a sequence of operator-valued random variables is defined and proved to converge in distribution to a random variable of which distribution is the proposed generalized Wiener measure, i.e., the generalization of the Wiener measure on $\{C([0,1], R), \mathcal{C}\}$. Section 3 concerns with weak convergence corresponding to the cadlag operator defined in this article. In Section 3.1, a metric for the space of cadlag operators is defined. Weak convergence of a sequence of operator-valued random variables on the metric space to a random variable of which distribution is the proposed generalized Poisson measure, i.e., the generalization of the Poisson measure on $\{D([0,1], R), \mathcal{D}\}$, is proved. Moreover, another metric for the subspace of the metric space in Section 3.1 is defined in Section 3.2 and the results concerning both completeness and separability of the corresponding metric space are given. Thus, the sufficient conditions analogous to the ones in the Polish space of continuous operators are established for weak convergence of a sequence of probability measures on the Polish space of cadlag operators. Since additional lemmas might be required for some theorems and the proofs are long, the proofs of these theorems in Section 2 and Section 3 are given in Section 4 and Section 5, respectively. Note that the extended results for the space of cadlag operators on some unbounded set along with the proofs of some theorems and lemmas are delegated to the supplementary materials, which can be found at http://web.thu .edu.tw/wenwei/www/papers/bnSupplement2016.pdf/

Hereafter, let $(\Omega, \Sigma, P)$ be the probability space on which the random variables of interest are defined, where $\Omega$ is a sample space, $\Sigma$ is the $\sigma$-field of 
Borel subsets of $\Omega$, and $P$ is a probability measure on $(\Omega, \Sigma)$. Furthermore, $P f^{-1}$, the induced probability measure on $\left(\Omega^{*}, \Sigma^{*}\right)$, is defined by $P f^{-1}(A)=$ $P(\{x: f(x) \in A\})$, where $f: \Omega \rightarrow \Omega^{*}$ is a Borel measurable function and $A \in \Sigma^{*}$. As $\Omega$ has been specified, the probability measure $P$ on $\Sigma$ rather than on $(\Omega, \Sigma)$ is used for succinctness. The notation $\|\cdot\|_{Z}$ is denoted as the norm of the normed space $Z$. The metric space with the metric $d$ induced by the norm is $d\left(z_{1}, z_{2}\right)=\left\|z_{1}-z_{2}\right\|_{Z}$ for $z_{1}, z_{2} \in Z$. Two results have been extensively used in the proofs. One is Theorem 2.7 of [4] referred to as the mapping theorem while the other is the lemma given in Section 8.1 of [7] referred to as the converging together lemma.

\section{Weak Convergence of Probability Measures on Metric Space of Continuous Operators}

Let $K$ be a compact set of the real separable Banach space $X$ and contain zero element. Since a compact subset of any metric space is bounded, $K$ is bounded. Assume that the range of the norm function on $K$ is $[0,1]$, i.e., $0 \leq\|x\|_{X} \leq 1, x \in K$. Denote $B(a, r)$ and $\bar{B}(a, r)$ as the open ball and closed ball with the center $a$ and the radius $r$ in a metric space, respectively. Let $F: K \rightarrow Y$ be the continuous operator with the domain $K$ and $\mathcal{Y}$ be the Borel $\sigma$-field of subsets of $Y$. Note that $F$ is also bounded. The metric space $[C(K, Y), \rho]$ of the $Y$-valued continuous operators defined on $K$ with the metric

$$
\rho\left(F_{1}, F_{2}\right)=\sup _{x \in K}\left\|F_{1}(x)-F_{2}(x)\right\|_{Y},
$$

is of interest, where $F_{1}, F_{2} \in[C(K, Y), \rho]$. Let $[\mathcal{C}(K, Y), \rho]$ be the Borel $\sigma$ field generated by the open sets of $C(K, Y)$. Also, let $\pi_{x_{1} \cdots x_{k}}: C(K, Y) \rightarrow Y^{k}$ be the natural projection defined by $\pi_{x_{1} \cdots x_{k}}(F)=\left[F\left(x_{1}\right), \ldots, F\left(x_{k}\right)\right]$ and $\mathcal{Y}^{k}$ be the Borel $\sigma$-field or product $\sigma$-field (see [3], Theorem 4.43) of subsets of the product space $Y^{k}$, where $x_{1}, \ldots, x_{k} \in K$. Note that $\pi_{x}$ is continuous and thus Borel measurable. In addition, $Y$ and $Y^{k}$ are both metric spaces with the metrics induced by $\|\cdot\|_{Y}$ and $\|\cdot\|_{Y^{k}}$, respectively. The metric space $[C(K, Y), \rho]$ is separable and complete, i.e., a Polish space, by Lemma 3.83 and Lemma 3.85 of [3]. For a $Y$-valued Radon Gaussian variable $g$, let

$$
\Sigma(g)=\sup _{\|T\|_{Y^{*}} \leq 1, T \in Y^{*}}\left\{E\left\{[T(g)]^{2}\right\}\right\}^{1 / 2},
$$


where $Y^{*}$ is the topological dual space of $Y$. In the following, the proposed generalized Wiener measure is defined first.

Definition 2.1. A generalized Wiener measure $W_{\phi}$, a probability measure on the space $[\mathcal{C}(K, Y), \rho]$, is the distribution of a $C(K, Y)$-valued random variable $\mathcal{W}_{\phi}$ with the following properties:

(i) $\phi: K \rightarrow[0,1]$ is a function with its range equal to the unit interval $[0,1]$ and $\phi(x)=0$ if and only if $x=0$.

(ii) $W_{\phi}(\{F: F \in C(K, Y), F(0)=0\})=1$.

(iii) The random variable $\mathcal{W}_{\phi}(x)=\pi_{x}\left(\mathcal{W}_{\phi}\right)$ for $x \neq 0$ under $W_{\phi}$ is a $Y$ valued centered Radon Gaussian variable. In other words, for any continuous linear functional $T \in Y^{*}$ on $Y, T\left[\mathcal{W}_{\phi}(x)\right]$ is a real-valued Gaussian variable with mean 0. Furthermore,

$$
\Sigma\left[\mathcal{W}_{\phi}(x)\right]=\sup _{\|T\|_{Y^{*}} \leq 1, T \in Y^{*}}\left\{E\left\{\left\{T\left[\mathcal{W}_{\phi}(x)\right]\right\}^{2}\right\}\right\}^{1 / 2}=[\phi(x)]^{1 / 2}
$$

(iv) For $0 \leq \phi\left(x_{0}\right) \leq \phi\left(x_{1}\right) \leq \cdots \leq \phi\left(x_{k}\right) \leq 1$, the random variables $\mathcal{W}_{\phi}\left(x_{1}\right)-\mathcal{W}_{\phi}\left(x_{0}\right), \mathcal{W}_{\phi}\left(x_{2}\right)-\mathcal{W}_{\phi}\left(x_{1}\right), \ldots, \mathcal{W}_{\phi}\left(x_{k}\right)-\mathcal{W}_{\phi}\left(x_{k-1}\right)$ are independent under $W_{\phi}$.

The generalized Wiener measure can be constructed based on the following results. First, the following theorem establishes the sufficient conditions for the tightness of a sequence of probability measures $\left\{P_{n}\right\}$ on $[\mathcal{C}(K, Y), \rho]$, which is one of main conditions for weak convergence of $\left\{P_{n}\right\}$. Let

$$
w(F, \Delta)=\sup _{\left\|x_{1}-x_{2}\right\|_{X} \leq \Delta}\left\|F\left(x_{1}\right)-F\left(x_{2}\right)\right\|_{Y}
$$

for $F \in[C(K, Y), \rho]$. Note that the function $w(\cdot, \Delta)$ on $[C(K, Y), \rho]$ is continuous for fixed $\Delta$ and hence measurable with respect to $[\mathcal{C}(K, Y), \rho]$ and $\mathcal{R}$, where $\mathcal{R}$ is the Borel $\sigma$-field of subsets of $R$. Let $K^{*}$ be a countable dense subset of $K$.

Theorem 2.1. If $\left\{P_{n} \pi_{x_{i}}^{-1}\right\}$ is relatively compact for each $x_{i} \in K^{*}$ and there exist $a \Delta$ and an $n_{0}$ such that

$$
P_{n}\left(\left\{F: w(F, \Delta) \geq \epsilon_{1}\right\}\right) \leq \epsilon_{2}, n \geq n_{0},
$$


for every positive $\epsilon_{1}$ and $\epsilon_{2}$, then the sequence of probability measures $\left\{P_{n}\right\}$ on the metric space $[\mathcal{C}(K, Y), \rho]$ is tight.

The following two theorems give the sufficient conditions for weak convergence of the sequence of probability measures $\left\{P_{n}\right\}$ and the convergence of a sequence of $C(K, Y)$-valued random variables $\mathcal{F}_{n}$ in distribution to a $C(K, Y)$-valued random variable $\mathcal{F}$. The notation $\Rightarrow$ denotes weak convergence or convergence in distribution.

Theorem 2.2. If $P_{n}$ is tight and $P_{n} \pi_{x_{1} \cdots x_{k}}^{-1} \Rightarrow Q_{x_{1} \cdots x_{k}}$ for all $x_{1}, \ldots, x_{k}$ in $K$, then there exists a probability measure $P$ on the space $[\mathcal{C}(K, Y), \rho]$ with $P \pi_{x_{1} \cdots x_{k}}^{-1}=Q_{x_{1} \cdots x_{k}}$ such that the sequence of probability measures $\left\{P_{n}\right\}$ on $[\mathcal{C}(K, Y), \rho]$ converges weakly to $P$, where $Q_{x_{1} \cdots x_{k}}$ is a probability measure on $\left(Y^{k}, \mathcal{Y}^{k}\right)$.

As $P$ is known, a direct result based on Theorem 2.2 is given below. Note that weak convergence of the finite dimensional distributions of $\left\{P_{n}\right\}$ to a probability measure $P$ in a set holds if $P_{n} \pi_{x_{1} \cdots x_{k}}^{-1} \Rightarrow P \pi_{x_{1} \cdots x_{k}}^{-1}$ for all $x_{1}, \ldots, x_{k}$ in the set.

Corollary 2.1. Let $\left\{P_{n}\right\}$ and $P$ be probability measures on the space $[\mathcal{C}(K, Y), \rho]$. If $\left\{P_{n}\right\}$ is tight and weak convergence of the finite dimensional distributions of $\left\{P_{n}\right\}$ to $P$ in $K$ holds, then $\left\{P_{n}\right\}$ converges weakly to $P$.

The convergence of a sequence of $C(K, Y)$-valued random variables $\mathcal{F}_{n}$ in distribution to a $C(K, Y)$-valued random variable $\mathcal{F}$ can be established based on the above theorems. Let the random vector

$$
\pi_{x_{1} \cdots x_{k}}(\mathcal{F})=\left[\mathcal{F}\left(x_{1}\right), \ldots, \mathcal{F}\left(x_{k}\right)\right],
$$

where $\mathcal{F}\left(x_{i}\right)$ is the random variable with the value equal to the projection of $\mathcal{F}$ at $x_{i}$ and at every sample point.

Theorem 2.3. If

$$
\lim _{\Delta \rightarrow 0} \limsup _{n \rightarrow \infty} P\left[w\left(\mathcal{F}_{n}, \Delta\right) \geq \epsilon\right]=0,
$$

for each positive $\epsilon$ and $\left\{\pi_{x_{1} \cdots x_{k}}\left(\mathcal{F}_{n}\right)\right\}$ converges in distribution to $\mathcal{G}_{x_{1} \cdots x_{k}}$ for all $x_{1}, \ldots, x_{k}$ in $K$, then there exists a $C(K, Y)$-valued random variable $\mathcal{F}$ with the distribution of $\pi_{x_{1} \cdots x_{k}}(\mathcal{F})$ equal to the one of the $Y^{k}$-valued random 
variable $\mathcal{G}_{x_{1} \cdots x_{k}}$ such that the sequence of $C(K, Y)$-valued random variables $\left\{\mathcal{F}_{n}\right\}$ converges in distribution to the $C(K, Y)$-valued random variable $\mathcal{F}$.

Proof. Let $P_{\mathcal{F}_{n}}$ be the distribution of $\mathcal{F}_{n}$. By the equation and Theorem 2.1 then, $\left\{P_{\mathcal{F}_{n}}\right\}$ is tight. By the convergence of $\left\{\pi_{x_{1} \cdots x_{k}}\left(\mathcal{F}_{n}\right)\right\}$ in distribution to $\mathcal{G}_{x_{1} \cdots x_{k}}$ for all $x_{1}, \ldots, x_{k}$ in $K$ and tightness of $\left\{P_{\mathcal{F}_{n}}\right\}$, the result follows by Theorem 2.2 .

The equation in the above theorem, the counterpart of the one given in Theorem 2.1, is the key for the tightness of the corresponding sequence of probability measures. The following corollary, a direct result by Theorem 2.3 , is a generalization of Theorem 7.5 of [4]. Note that the convergence of the finite dimensional distributions of $\mathcal{F}_{n}$ to $\mathcal{F}$ in $K$ holds if $\left\{\pi_{x_{1} \cdots x_{k}}\left(\mathcal{F}_{n}\right)\right\}$ converges in distribution to $\pi_{x_{1} \cdots x_{k}}(\mathcal{F})$ for all $x_{1}, \ldots, x_{k}$ in $K$.

Corollary 2.2. Let $\left\{\mathcal{F}_{n}\right\}$ and $\mathcal{F}$ be $C(K, Y)$-valued random variables. If

$$
\lim _{\Delta \rightarrow 0} \limsup _{n \rightarrow \infty} P\left[w\left(\mathcal{F}_{n}, \Delta\right) \geq \epsilon\right]=0,
$$

for each positive $\epsilon$ and the convergence of the finite dimensional distributions of $\mathcal{F}_{n}$ to $\mathcal{F}$ in $K$ holds, then $\left\{\mathcal{F}_{n}\right\}$ converges in distribution to $\mathcal{F}$.

Based on the above results, a sequence of random variables can be constructed and its limiting distribution is the generalized Wiener measure. Consider a $C(K, Y)$-valued random variable $\mathcal{F}_{n}$ defined by

$$
\mathcal{F}_{n}(x, w)=\frac{S_{\lfloor n \phi(x)\rfloor}(w)}{\sqrt{n}}+\frac{c_{n}(x) \xi_{\lfloor n \phi(x)\rfloor+1}(w)}{\sqrt{n}},
$$

for $w \in \Omega$, where $\phi$ is the function given in (i) of Definition 2.1 and assumed to be continuous, $c_{n}(x)=n \phi(x)-\lfloor n \phi(x)\rfloor, S_{\lfloor n \phi(x)\rfloor}=\sum_{i=1}^{\lfloor n \phi(x)\rfloor} \xi_{i}, S_{0}=0$, $\xi_{i}$ are independent copies of a $Y$-valued random variables $\xi$, and $\lfloor\cdot\rfloor$ is the Gauss's floor function. Given the conditions related to the size of the net for $K$, the existence of the generalized Wiener measure can be proved and the generalization of Donsker's theorem can be obtained, as indicated by the following theorem. One difference between (a) and (b) of the theorem is the assumptions imposed on $\xi$, one being centered Radon Gaussian and the other satisfying the central limit theorem (CLT), i.e., $\sum_{i=1}^{n} \xi_{i} / n^{1 / 2}$ converging weakly in the Banach space $Y$ (see [10], Chapter 10). Since the proof of 
(b) is analogous to the one of (a), the proof is delegated to the supplementary materials.

Theorem 2.4. Let the function $\phi$ satisfy the Lipschitz condition, i.e., there exists a constant $L$ such that $\left|\phi\left(x_{1}\right)-\phi\left(x_{2}\right)\right| \leq L|| x_{1}-x_{2} \|_{X}$ for $x_{1}, x_{2} \in K$.

(a) Suppose that $Y$ is a cotype 2 Banach space (see [10, Chap. 9]) and $\xi$ is a centered Radon Gaussian variable with $\Sigma(\xi)=1$. If there exists a positive number $\delta$ such that for a $\Delta$-net for $K, \Delta<\delta$, the number of points in the net, $v$, satisfies the inequality $v \leq h\left(\Delta^{-1}\right) \Delta^{-1}$, then there exists a probability measure $W_{\phi}$ on the metric space $[\mathcal{C}(K, Y), \rho]$ as given in Definition 2.1, where $h$ is an increasing function, $h(n)=o\left[n^{-1} \exp (\right.$ an $\left.)\right]$ for any positive number $a>0$, and where $n$ is a positive number.

(b) Suppose that the symmetric $\xi$ satisfies $\sum_{i=1}^{n} \xi_{i} / \sqrt{n} \Rightarrow \mathcal{W}$, i.e., $\xi$ satisfying CLT, where $\mathcal{W}$ is a $Y$-valued centered Radon Gaussian variable with $\Sigma(\mathcal{W})=1$. If there exists a positive number $\delta$ and a positive number $C$ such that for a $\Delta$-net for $K, \Delta<\delta$, the number of points in the net, $v$, satisfies the inequality $v \leq C \Delta^{-1}$, then the sequence of $C(K, Y)$-valued random variables $\left\{\mathcal{F}_{n}\right\}$ converges in distribution to the random variable $\mathcal{W}_{\phi}$

\section{Weak Convergence of Probability Measures on Metric Spaces of Cadlag Operators}

The results in Section 2 are mainly for continuous random operators. However, the space $[C(K, Y), \rho]$ might not be suitable for Poisson-type random operators, i.e, the cadlag random operators. The generalizations of several results given in Section 12, Section 13 and Section 16 of [4] and [11] are given in this section and supplementary materials. In Section 3.1 and Section 3.2, the cadlag operators on compact domains are of interest, while the ones on an unbounded domain are considered in the supplementary materials. In Section 3.1, the generalized Poisson measure is defined and a sequence of random variables is constructed and proved to converge in distribution to an operator-valued random variable having the generalized Poisson measure as its distribution. In Section 3.2, a metric playing the role analogous to the Skorohod metric on $D[0,1]$ is defined and the associated space of cadlag operators is proved to be Polish. Then, the sufficient 
conditions for the tightness of a sequence of probability measures and weak convergence of the sequence of probability measures are established. Moreover, the results given in Section 3.2 are extended to cadlag operators on the unbounded domain in the supplementary materials. The relation $<$ in the subset $S$ of $X$ is defined by $x_{1}<x_{2}$ provided that $\left\|x_{1}\right\|_{X}<\left\|x_{2}\right\|_{X}$. Note that the relation is a linear order in terms of the equivalence class $[c]=\left\{x:\|x\|_{X}=c, x \in S\right\}$, where $c \in R$ and the equivalence relation is the equality of the normed values. In this section, the relation is used for the elements of the subsets of $X$. The cadlag operator of interest is defined first.

Definition 3.1. Let $S$ be a bounded subset of $X$ and there exist elements $\underline{x}$ and $\bar{x}$ in $S$ such that $\|\underline{x}\|_{X}=\inf _{x \in S}\|x\|_{X}$ and $\|\bar{x}\|_{X}=\sup _{x \in S}\|x\|_{X}$. The cadlag $Y$-valued operator defined on $S$ has the following properties:

(i) The right-hand limit at any $x_{0}$ in $S \cap\left\{x:\|x\|_{X}=\|\bar{x}\|_{X}\right\}^{c}$ exists and is equal to $F\left(x_{0}\right)$, i.e., for every $\epsilon>0$ there is a $\delta>0$ such that $\| F(x)-$ $F\left(x_{0}\right) \|_{Y}<\epsilon$ for $x \in S, x>x_{0}$, and $\left\|x-x_{0}\right\|_{X}<\delta$. The notation is defined by

$$
F\left(x_{0}\right)=F\left(x_{0}^{+}\right)=\lim _{x \rightarrow x_{0}, x>x_{0}} F(x) .
$$

(ii) The left-hand limit at any $x_{0}$ in $S \cap\left\{x:\|x\|_{X}=\|\underline{x}\|_{X}\right\}^{c}$ exists, i.e., for every $\epsilon>0$ there is a $\delta>0$ and a $y_{0} \in Y$ such that $\left\|F(x)-y_{0}\right\|_{Y}<\epsilon$ for $x \in S, x<x_{0}$, and $\left\|x-x_{0}\right\|_{X}<\delta$. The notation is defined by

$$
y_{0}=F\left(x_{0}^{-}\right)=\lim _{x \rightarrow x_{0}, x<x_{0}} F(x)
$$

The above $Y$-valued operator is right-continuous and has left-hand limit on $S$. The sum $F_{1}+F_{2}$ of two cadlag operators $F_{1}$ and $F_{2}$ on $S$ is

$$
\left(F_{1}+F_{2}\right)(x)=F_{1}(x)+F_{2}(x)
$$

and the scalar product $\alpha F$ of the cadlag operator $F$ on $S$ is

$$
(\alpha F)(x)=\alpha F(x)
$$

for $x \in S$, where $\alpha \in R$. 


\subsection{Generalized Poisson measure}

The compact set $\tilde{K}_{1} \subset X$ of interest in this and next subsections has the following property: $\tilde{K}_{1} \neq\{0\},\left\{x: x=c x_{0} /\left\|x_{0}\right\|_{X}, 0 \leq c \leq 1\right\} \subset \tilde{K}_{1}$ for any $x_{0} \neq 0$ in $\tilde{K}_{1}$, and $0<\left\|x_{0}\right\|_{X} \leq 1$. The equivalence classes of interest are $[c]=\left\{x:\|x\|_{X}=c, x \in \tilde{K}_{1}\right\}$, where $0 \leq c \leq 1$.

In the following, the space of the cadlag $Y$-valued operators is introduced. Then, both the Skorohod metric imposed on the space and the generalized Poisson measure can be defined on the associated measure space.

Definition 3.2. Let $D\left(\tilde{K}_{1}, Y\right)$ be the space (or set) of the cadlag $Y$-valued operators defined on $\tilde{K}_{1}$ with the following properties:

(i) $F$ is bounded, i.e., $\|F\|_{\text {sup }}=\sup _{x \in \tilde{K}_{1}}\|F(x)\|_{Y}<\infty$ for $F \in D\left(\tilde{K}_{1}, Y\right)$.

(ii) $\left\{F(x):\|x\|_{X}=1, x \in \tilde{K}_{1}\right\}$ is totally bounded for $F \in D\left(\tilde{K}_{1}, Y\right)$.

Denote

$$
w_{1}\left(F, \tilde{S}_{1}\right)=\sup _{x, x^{*} \in \tilde{S}_{1}}\left\|F(x)-F\left(x^{*}\right)\right\|_{Y}
$$

where $\tilde{S}_{1}$ is any subset of $\tilde{K}_{1}$. In addition, let

and

$$
w_{1}^{\prime}(F, \Delta)=\inf _{\left\{s_{i}\right\}} \max _{i} w_{1}\left\{F,\left[s_{i-1}, s_{i}\right)\right\}
$$

$$
w_{1}^{\prime}(F, \Delta, x)=\inf _{\left\{s_{i}\right\}} \max _{i} w_{1}\left\{F,\left[s_{i-1}, s_{i}\right)_{x}\right\}
$$

where $\left\{s_{i}\right\}$, called $\Delta$-sparse, is any sequence satisfying $0=s_{0}<s_{1}<\cdots<$ $s_{k}=1$ and $\min _{1 \leq i \leq k}\left(s_{i}-s_{i-1}\right)>\Delta,[a, b)=\left\{x: a \leq\|x\|_{X}<b, x \in \tilde{K}_{1}\right\}$, $[a, b)_{x}=\left\{c x /\|x\|_{X}: a \leq c<b\right\}$ for $x \in \tilde{K}_{1}$, and $0 \leq a<b \leq 1$. Hereafter the interval notation implicitly corresponds to the one in $X$ and the interval of real numbers will be described explicitly.

In the following, the Skorohod metric for the cadlag random operators is defined first. The Poisson convergence, the generalized Poisson measure, as weak convergence of a sequence of probability measures, is given then.

Definition 3.3. Denote $\Lambda_{1}$ as a class of functions $\lambda$ defined on $\tilde{K}_{1}$ with the following properties: 
(i) $\lambda: \tilde{K}_{1} \rightarrow \tilde{K}_{1}$ mapping $[0,1]_{x}$ onto $[0,1]_{x}$ for $x \in \tilde{K}_{1}$, is a strictly increasing function in terms of paths, i.e., $\lambda\left(x_{1}\right)>\lambda\left(x_{2}\right)$ if $x_{1}>x_{2}, x_{1}, x_{2} \in$ $[0,1]_{x}$, and a continuous function.

(ii) $\lambda$ has an inverse $\lambda^{-1}$.

(iii) $\lambda(x)=x$ as $\|x\|_{X}=1$.

The Skorohod function on $D\left(\tilde{K}_{1}, Y\right) \times D\left(\tilde{K}_{1}, Y\right)$ corresponding to the class $\Lambda_{1}$ is defined by

$$
d_{1}\left(F_{1}, F_{2}\right)=\inf _{\lambda \in \Lambda_{1}} \max \left(\|\lambda-I\|_{\text {sup }},\left\|F_{1}-F_{2} \lambda\right\|_{\text {sup }}\right)
$$

for $F_{1}, F_{2} \in D\left(\tilde{K}_{1}, Y\right)$, where $I$ is the identity map,

$$
\|\lambda-I\|_{\text {sup }}=\sup _{x \in \tilde{K}_{1}}\|\lambda(x)-x\|_{X}
$$

and $F_{2} \lambda$ is the composition of the operator $F_{2}$ and the function $\lambda$.

The metric $\rho$ corresponding to the norm is defined by $\rho\left(F_{1}, F_{2}\right)=\| F_{1}-$ $F_{2}\left\|_{\text {sup }}=\sup _{x \in K}\right\| F_{1}(x)-F_{2}(x) \|_{Y}$ on the space $C(K, Y)$, where $F_{1}, F_{2} \in$ $C(K, Y)$. However, the Skorohod metric can not be induced by the norm on the space $D\left(\tilde{K}_{1}, Y\right)$. Note that $\left[D\left(\tilde{K}_{1}, Y\right), d_{1}\right]$ being a metric space and the measurability of the projection operator $\pi_{x_{1} \ldots x_{k}}$, given and proved in Section 5 , are required to define the generalized Poisson measure, where $x_{1}, \ldots, x_{k} \in$ $\tilde{K}_{1}$. Let $\left[\mathcal{D}\left(\tilde{K}_{1}, Y\right), d_{1}\right]$ be the Borel $\sigma$-field of subsets of $\left[D\left(\tilde{K}_{1}, Y\right), d_{1}\right]$.

Definition 3.4. A generalized Poisson measure $P_{\phi, \alpha}$, a probability measure on the metric space $\left\{\left[D\left(\tilde{K}_{1}, Y\right), d_{1}\right],\left[\mathcal{D}\left(\tilde{K}_{1}, Y\right), d_{1}\right]\right\}$, is the distribution of a $D\left(\tilde{K}_{1}, Y\right)$-valued random variable $\mathcal{F}$ with the following properties:

(i) $\phi: \tilde{K}_{1} \rightarrow[0,1]$ is a function with its range equal to the unit interval $[0,1]$ of real numbers and $\phi(x)=0$ if and only if $x=0$.

(ii) $P_{\phi, \alpha}\left(\left\{F: F \in\left[D\left(\tilde{K}_{1}, Y\right), d_{1}\right], F(0)=0\right\}\right)=1$.

(iii) The random variable $\mathcal{F}(x)=\pi_{x}(\mathcal{F})$ for $x \neq 0$ under $P_{\phi, \alpha}$ is a $Y$-valued Poisson variable with mean $\alpha \phi(x)$, i.e.,

$$
P_{\phi, \alpha}\left(\left\{F: F \in D\left(\tilde{K}_{1}, Y\right), F(x) \in A_{i}\right\}\right)=e^{-\alpha \phi(x)} \frac{[\alpha \phi(x)]^{i}}{i !}, i=0,1,2, \ldots,
$$

and

$P_{\phi, \alpha}\left(\left\{F: F \in D\left(\tilde{K}_{1}, Y\right), F(x) \in A_{i}^{*}\right\}\right)=0$, 
where $A_{i}=\left\{z:\|z\|_{Y}=i, z \in Y\right\}$ and $A_{i}^{*} \in \mathcal{Y}$ is any proper measurable subset of $A_{i}$.

(iv) For $0 \leq \phi\left(x_{0}\right) \leq \phi\left(x_{1}\right) \leq \cdots<\phi\left(x_{k}\right) \leq 1$, the random variables $\mathcal{F}\left(x_{1}\right)-\mathcal{F}\left(x_{0}\right), \mathcal{F}\left(x_{2}\right)-\mathcal{F}\left(x_{1}\right), \ldots, \mathcal{F}\left(x_{k}\right)-\mathcal{F}\left(x_{k-1}\right)$ are independent under $P_{\phi, \alpha}$.

As $Y=R, \tilde{K}_{1}=[0,1]$, and $\phi(x)=x$, the generalized Poisson measure is the Poisson measure on the real-valued Poisson process. The generalized Poisson measure depends on one more "parameter" $\phi$ than the Poisson measure. Since the set $A_{i}$ might have multiple sample points, there may exist two different probability measures satisfying the first equation given in condition (iii), but may assign different probabilities corresponding to the subsets of $A_{i}$. The possibility can be excluded by the second equation in condition (iii).

The proof of next theorem is analogous to Theorem 12.6 of [4]. By imbedding a set with probability one in $Y^{\infty}=Y \times Y \times \cdots$ endowed with a certain metric topology and using the Portmanteau theorem (see [4], Theorem 2.1), the result of weak convergence can be obtained then. Let $\tilde{K}_{1 c}$ be a countable dense set of $\tilde{K}_{1}$.

Theorem 3.1. If there exists a set $E \in\left[\mathcal{D}\left(\tilde{K}_{1}, Y\right), d_{1}\right]$ satisfying that $P(E)=$ $P_{n}(E)=1, F_{n}(x) \underset{n \rightarrow \infty}{\longrightarrow} F(x)$ for all $x$ in $\tilde{K}_{1 c}$ implies $F_{n} \underset{n \rightarrow \infty}{\longrightarrow} F$ in the Skorohod topology for $F, F_{n} \in E$, and weak convergence of the finite dimensional distributions of $\left\{P_{n}\right\}$ to $P$ in $\tilde{K}_{1 c}$ holds, then the sequence of probability measures $\left\{P_{n}\right\}$ converges weakly to the probability measure $P$.

Proof. The metric in $Y^{\infty}$ is defined by

$$
\rho\left(y_{1}^{\infty}, y_{2}^{\infty}\right)=\sum_{i=1}^{\infty} \frac{a^{-i} \rho^{*}\left(y_{1 i}, y_{2 i}\right)}{b+\rho^{*}\left(y_{1 i}, y_{2 i}\right)}
$$

for $y_{1}^{\infty}=\left(y_{11}, y_{12}, \ldots\right), y_{2}^{\infty}=\left(y_{21}, y_{22}, \ldots\right)$ in $Y^{\infty}$, where $a>1, b>0$, and $\rho^{*}\left(y_{1 i}, y_{2 i}\right)=\left\|y_{1 i}-y_{2 i}\right\|_{Y}$. Let $\tilde{K}_{1 c}=\left\{x_{i}: i=1,2, \ldots\right\}$ and $\pi$ : $D\left(\tilde{K}_{1}, Y\right) \rightarrow Y^{\infty}$ defined by $\pi(F)=\left[F\left(x_{1}\right), F\left(x_{2}\right), \ldots\right]$ and $\pi_{k}: Y^{\infty} \rightarrow Y^{k}$ by $\pi_{k}\left(y_{1}^{\infty}\right)=\left(y_{11}, y_{12}, \ldots, y_{1 k}\right)$.

Next is to prove the measurability of $\pi$ which can imbed the set of $D\left(\tilde{K}_{1}, Y\right)$ into $Y^{\infty}$. Since $Y$ is a Polish space, $Y^{\infty}$ is second-countable and 
hence separable by Theorem 30.2 of [12]. Further, because $\rho\left(y_{n}, z\right) \underset{n \rightarrow \infty}{\longrightarrow} 0$ implies $\rho^{*}\left(y_{n i}, z_{i}\right) \underset{n \rightarrow \infty}{\longrightarrow} 0, \pi_{k}$ is continuous and hence Borel measurable. Let the ball (Borel) $\sigma$-field of subsets of $Y^{\infty}$ be $\mathcal{Y}^{\infty}$ and let the class of finitedimensional sets i.e., the sets of the form $\pi_{k}^{-1}(S), S \subset \mathcal{Y}^{k}$, be $\mathcal{Y}_{f}^{\infty}$. Since $\pi_{k}$ is Borel measurable, $\mathcal{Y}_{f}^{\infty} \subset \mathcal{Y}^{\infty}$. Note that $\mathcal{Y}_{f}^{\infty}$ is a $\pi$ system and there exist open subsets in $\mathcal{Y}_{f}^{\infty}$ satisfying

$N\left(y_{1}^{\infty}, r^{*}, k\right)=\left\{y_{2}^{\infty}:\left\|y_{2 i}-y_{1 i}\right\|_{Y}<r^{*}, i=1, \ldots, k, y_{2}^{\infty} \in Y^{\infty}\right\} \subset B\left(y_{1}^{\infty}, r\right)$,

i.e., $\left\{N\left(y_{1}^{\infty}, r^{*}, k\right)\right\}$ being a base for the topology of $Y^{\infty}$, where $y_{1}^{\infty}$ is any element in $Y^{\infty}, r>0$, and $r^{*}<b\left[(a-1) r-a^{-k}\right]$ by choosing some value of $k$. Therefore, $\mathcal{Y}_{f}^{\infty}$ generates the Borel $\sigma$-field $\mathcal{Y}^{\infty}$ and is a separating class of $\mathcal{Y}^{\infty}$ (see [4], p. 9). This gives that $\pi$ is Borel measurable owing to $\pi^{-1}\left[\pi_{k}^{-1}(S)\right]=\pi_{x_{1} \ldots x_{k}}^{-1}(S)$.

Finally, because for each $y_{1}^{\infty}$ and $r, N\left(y_{1}^{\infty}, r^{*}, k\right) \subset B\left(y_{1}^{\infty}, r\right), Y^{\infty}$ is separable, and the class of boundaries of $N\left(y_{1}^{\infty}, r^{*}, k\right), r^{*}<b\left[(a-1) r-a^{-k}\right]$, contains uncountably many disjoint sets, $\mathcal{Y}_{f}^{\infty}$ is a convergence-determining class (see [4], p. 18) of $\mathcal{Y}^{\infty}$ by Theorem 2.4 of [4]. Thus, since weak convergence of the finite dimensional distributions of $\left\{P_{n}\right\}$ to $P$ in $\tilde{K}_{1 c}$ holds, $P_{n} \pi^{-1} \Rightarrow P \pi^{-1}$ by the fact that $\mathcal{Y}_{f}^{\infty}$ is both the separating and convergencedetermining class of $\mathcal{Y}^{\infty}$. The proof then follows along with the lines given in the second paragraph of the proof of Theorem 12.6 of [4] by replacing $R^{\infty}$ with $Y^{\infty}$ and $(D, \mathcal{D})$ with $\left\{\left[D\left(\tilde{K}_{1}, Y\right), d_{1}\right],\left[\mathcal{D}\left(\tilde{K}_{1}, Y\right), d_{1}\right]\right\}$.

Note that the conditions $P(E)=P_{n}(E)=1$ and pointwise convergence implying convergence in the Skorohod topology play the similar role to the tightness of $\left\{P_{n}\right\}$. For a real-valued Poisson process, it is a limit of a sequence of random functions based on the sum of Bernoulli random variables (see [4], Example 12.3). Analogous result follows for a sequence of random operators defined as follows. For each $n, x \in \tilde{K}_{1}$, and $w \in \Omega$,

$$
\mathcal{F}_{n}(x, w)=\sum_{j \leq\lfloor n \phi(x)\rfloor} \xi_{n j}(w)
$$

as $x \notin[1]$ and

$$
\mathcal{F}_{n}(x, w)=\sum_{j \leq(n-1)} \xi_{n j}(w)
$$


as $x \in[1]$, where $\xi_{n j}, j=1,2, \ldots$, are independent copies of a $Y$-valued Bernoulli random variables $\xi$ taking values $y$ and 0 with probabilities $\alpha / n$ and $1-\alpha / n$, respectively, $\phi(x)=\|x\|_{X}$, and where $\|y\|_{Y}=1$. Let $z_{i}=$ $i y, i=0,1, \ldots$. Note that the sample paths $F_{n}$ of $\mathcal{F}_{n}$ take values on $z_{i}$. The following corollary indicates the convergence of the sequence of random operators in distribution to the random element with the generalized Poisson measure as its distribution.

Corollary 3.1. Let $\mathcal{F}$ be the $D\left(\tilde{K}_{1}, Y\right)$-valued random variable having the following properties:

(i) The distribution of $\mathcal{F}$ is the generalized Poisson measure given in Definition 3.4 with $\phi(x)=\|x\|_{X}$ and $A_{i}$ consisting of only one point $z_{i}$.

(ii) The sample paths of $\mathcal{F}$ are nondecreasing operators which take values on $z_{i}$, take the same value at the points in the same equivalence class, and the jumps at the points of discontinuity are equal to 1, i.e., for $w \in \Omega, \mathcal{F}\left(x_{1}, w\right)=z_{i}, \mathcal{F}\left(x_{2}, w\right)=z_{j}, i \leq j, \mathcal{F}(x, w)=\mathcal{F}\left(x^{*}, w\right)$, and $\left\|\mathcal{F}\left(x_{d}, w\right)\right\|_{Y}-\left\|\mathcal{F}\left(x_{d}^{-}, w\right)\right\|_{Y}=1$ provided that $\left\|x_{1}\right\|_{X} \leq\left\|x_{2}\right\|_{X}$, $\|x\|_{X}=\left\|x^{*}\right\|_{X}$, and $\mathcal{F}(\cdot, w)$ is discontinuous at $x_{d}$.

(iii)

$$
P\left(\mathcal{F}(x)-\mathcal{F}\left(x^{-}\right) \neq 0, x \in[1]\right)=0 .
$$

Then, $\left\{\mathcal{F}_{n}\right\}$ converges in distribution to $\mathcal{F}$.

Proof. Let $E \in\left[\mathcal{D}\left(\tilde{K}_{1}, Y\right), d_{1}\right]$ and any $F$ in $E$ be a nondecreasing cadlag operator, have jumps of exactly 1 at its points of discontinuity, only take values on the set $\left\{z_{i}\right\}$ and take the same value at the points in the equivalence class, $F(0)=0$, and have no jump at [1]. Since all sample paths of $\mathcal{F}_{n}$ are in the set $E$ and $\mathcal{F}$ has properties (i), (ii), and (iii), the condition $P(E)=$ $P_{n}(E)=1$ in Theorem 3.1 holds. The point convergence for $F \in E$ implying the convergence in the Skorohod topology is proved as follows. Suppose that $F_{n}(x) \underset{n \rightarrow \infty}{\longrightarrow} F(x)$ for $x \in \tilde{K}_{1 c}$ and $F_{n}, F \in E$. Since there are at most finitely many equivalence classes at which the jumps $\left\|F(x)-F\left(x^{-}\right)\right\|_{Y}$ exceed a given positive number by the equation $\lim _{\Delta \rightarrow 0} w_{1}^{\prime}(F, \Delta)=0$ and the jump is $1, F$ has only finitely many discontinuity classes $\left[t_{1}\right], \ldots,\left[t_{k}\right]$ (see Remark 5.1 in Section 5.1). Let $K_{\epsilon i}=\left\{x: t_{i}-\epsilon \leq\|x\|_{X} \leq t_{i}+\epsilon\right\}$ for any given positive $\epsilon$, i.e., $x \in\left[t_{i}-\epsilon, t_{i}+\epsilon\right]$, and $t_{i}<1$. Then, for $n>n_{0}, F_{n}$ must have a single jump class in each $K_{\epsilon i}$ and agree with $F$ in $\tilde{K}_{1} \cap\left(\cup_{i} K_{\epsilon i}\right)^{c}$. Since 
$d_{1}\left(F_{n}, F\right)=d_{1}\left(F, F_{n}\right)$, it suffices to consider that the jump classes $\left[t_{n i}\right]$ of $F_{n}$ satisfy $t_{n i}>t_{i}$. Then, $F_{n}(x)=F(x)$ as $x \in K_{\epsilon i},\|x\|_{X}<t_{i}$, and $\|x\|_{X} \geq t_{n i}$. If

$\lambda_{n}(x)= \begin{cases}x_{a\left(t_{i}-\epsilon\right)}+\epsilon^{-1}\left\|x-x_{a\left(t_{i}-\epsilon\right)}\right\|_{X}\left(x_{a t_{n i}}-x_{a\left(t_{i}-\epsilon\right)}\right), & x \in\left[t_{i}-\epsilon, t_{i}\right)_{x_{a}} \\ x_{a t_{n i}}+\epsilon^{-1}\left\|x-x_{a t_{i}}\right\|_{X}\left(x_{a\left(t_{i}+\epsilon\right)}-x_{a t_{n i}}\right), & x \in\left[t_{i}, t_{i}+\epsilon\right]_{x_{a}}\end{cases}$

for $x \in\left[t_{i}-\epsilon, t_{i}+\epsilon\right]_{x_{a}}$ and every $x_{a}$ in $\tilde{K}_{1}$, then $\lambda_{n}\left(x_{a t_{i}}\right)=x_{a t_{n i}},\left\|\lambda_{n}-I\right\|_{s u p} \leq$ $2 \epsilon$, and $F_{n}\left[\lambda_{n}(x)\right]=F(x)$, where $x_{a t}=t x_{a} /\left\|x_{a}\right\|_{X}, 0 \leq t \leq 1$. Thus, $\left\{F_{n}\right\}$ converges to $F$ in the Skorohod topology. Therefore, the condition that pointwise convergence implies convergence in the Skorohod topology in Theorem 3.1 holds.

It remains to prove that weak convergence in terms of the finite-dimensional distributions, i.e., the last condition given in Theorem 3.1, holds. Since $\mathcal{F}_{n}(x)=\sum_{j \leq\lfloor n \phi(x)\rfloor} \xi_{n j}, x \notin[1]$, is a binomial random variables $B(\lfloor n \phi(x)\rfloor$, $\alpha / n)$ taking values on $z_{i}$, i.e.,

$$
P\left[\mathcal{F}_{n}(x)=z_{i}\right]=\left(\begin{array}{c}
\lfloor n \phi(x)\rfloor \\
i
\end{array}\right)\left(\frac{\alpha}{n}\right)^{i}\left(1-\frac{\alpha}{n}\right)^{\lfloor n \phi(x)\rfloor-i},
$$

$\left\{\mathcal{F}_{n}(x)\right\}$ converges in distribution to the random variable $\mathcal{F}(x)$ under $P_{\phi, \alpha}$ with $A_{i}=\left\{z_{i}\right\}$ by Poisson convergence and property (i) of $\mathcal{F}$. Note that the Poisson convergence also holds for $\mathcal{F}_{n}(x), x \in[1]$. Furthermore, because $\mathcal{F}_{n}\left(x_{1}\right)=\sum_{j \leq\left\lfloor n \phi\left(x_{1}\right)\right\rfloor} \xi_{n j}$ and $\mathcal{F}_{n}\left(x_{2}\right)-\mathcal{F}_{n}\left(x_{1}\right)=\sum_{\left\lfloor n \phi\left(x_{2}\right)\right\rfloor<j \leq\left\lfloor n \phi\left(x_{2}\right)\right\rfloor} \xi_{n j}$ are independent, $\left\{\left[\mathcal{F}_{n}\left(x_{1}\right), \mathcal{F}_{n}\left(x_{2}\right)-\mathcal{F}_{n}\left(x_{1}\right)\right]\right\}$ converges in distribution to $\left[\mathcal{F}\left(x_{1}\right), \mathcal{F}\left(x_{2}\right)-\mathcal{F}\left(x_{1}\right)\right]$ by the Poisson convergence and the mapping theorem, where $\mathcal{F}\left(x_{1}\right)$ and $\mathcal{F}\left(x_{2}\right)-\mathcal{F}\left(x_{1}\right)$ are independent under $P_{\phi, \alpha}$. Finally, $\left\{\left[\mathcal{F}_{n}\left(x_{1}\right), \mathcal{F}_{n}\left(x_{2}\right)\right]\right\}$ converges in distribution to $\left[\mathcal{F}\left(x_{1}\right), \mathcal{F}\left(x_{2}\right)\right]$ by employing the mapping theorem again. The convergence of $\left\{\left[\mathcal{F}_{n}\left(x_{1}\right), \ldots, \mathcal{F}_{n}\left(x_{k}\right)\right]\right\}$ in distribution to $\left[\mathcal{F}\left(x_{1}\right), \ldots, \mathcal{F}\left(x_{k}\right)\right]$ can be proved analogously.

\subsection{Cadlag operators with a compact domain}

The Poisson convergence is a special case of the general weak convergence theory on the space $D\left(\tilde{K}_{1}, Y\right)$. In the following, several theorems concerning the sufficient conditions for weak convergence of a sequence of 
probability measures on $D^{\circ}\left(\tilde{K}_{1}, Y\right)$, the subset of $D\left(\tilde{K}_{1}, Y\right)$, are given. Separability and completeness of the space $D^{\circ}\left(\tilde{K}_{1}, Y\right)$ in the sense of a suitable metric are crucial for the tightness of the sequence of probability measures, which is one of the sufficient conditions for weak convergence of the sequence of probability measures. However, the space $\left[D^{\circ}\left(\tilde{K}_{1}, Y\right), d_{1}\right]$ might not be complete. By defining another metric $d_{1}^{\circ}$, the space can be separable and complete as the space of real-valued cadlag functions on the unit interval $[0,1]$ of real numbers. The class of functions corresponding to the new metric is defined first.

Definition 3.5. Let $\Lambda_{1}^{\circ}$ be the subset of $\Lambda_{1}$ with property (i) given in Definition 3.3 replaced with the following property:

$(i)^{*} \lambda: \tilde{K}_{1} \rightarrow \tilde{K}_{1}$, mapping $[0,1]_{x}$ to $[0,1]_{x}$ for $x \in \tilde{K}_{1}$, is a strictly increasing function, i.e., $\lambda\left(x_{1}\right)>\lambda\left(x_{2}\right)$ if $x_{1}>x_{2}, x_{1}, x_{2} \in \tilde{K}_{1}$, and a continuous function.

Denote the function

$$
w_{1}^{\prime \prime}(F, \Delta)=\max \left\{w_{1}^{\prime}(F, \Delta), \max _{q} w_{1}\left[F, B^{*}\left(x_{q 1}, \Delta\right)\right]\right\},
$$

where $\left\{x_{q 1}\right\}$ is a $\Delta$-net for the equivalence class [1], $\left\{x_{q 1}\right\} \subset[1], B^{*}\left(x_{q 1}, \Delta\right) \subset$ $\left\{B\left(x_{q 1}, \Delta\right) \cap[1]\right\}, \cup_{q} B^{*}\left(x_{q 1}, \Delta\right)=[1]$, and the sets $B^{*}\left(x_{q 1}, \Delta\right)$ are disjoint.

Definition 3.6. Let $D^{\circ}\left(\tilde{K}_{1}, Y\right)$, the subset of $D\left(\tilde{K}_{1}, Y\right)$, have the following property: For $F \in D^{\circ}\left(\tilde{K}_{1}, Y\right)$, the equation

$$
\lim _{\Delta \rightarrow 0} w_{1}^{\prime \prime}(F, \Delta)=0
$$

holds.

In addition, the Skorohod functions on $D^{\circ}\left(\tilde{K}_{1}, Y\right) \times D^{\circ}\left(\tilde{K}_{1}, Y\right)$ corresponding to the class $\Lambda_{1}^{\circ}$ are defined by

$$
d_{1}^{*}\left(F_{1}, F_{2}\right)=\inf _{\lambda \in \Lambda_{1}^{\circ}} \max \left\{\|\lambda-I\|_{\text {sup }},\left\|F_{1}-F_{2} \lambda\right\|_{\text {sup }}\right\}
$$

and

$$
\begin{array}{r}
d_{1}^{\circ}\left(F_{1}, F_{2}\right)=\inf _{\lambda \in \Lambda_{1}^{\circ}} \max \left\{\sup _{x^{*}<x, x^{*}, x \in \tilde{K}_{1}}\left|\log \left[\frac{\|\lambda(x)\|_{X}-\left\|\lambda\left(x^{*}\right)\right\|_{X}}{\|x\|_{X}-\left\|x^{*}\right\|_{X}}\right]\right|,\right. \\
\left.\|\lambda-I\|_{\text {sup }},\left\|F_{1}-F_{2} \lambda\right\|_{\text {sup }}\right\}
\end{array}
$$


for $F_{1}, F_{2} \in D^{\circ}\left(\tilde{K}_{1}, Y\right)$.

Note that $d_{1}^{*}$ and $d_{1}^{\circ}$ are metrics. The routine proof of $d_{1}^{*}$ and $d_{1}^{\circ}$ being metrics is not presented. The function $w_{1}^{\prime \prime}$ plays the role similar to $w$ in Section 2. The function $w_{1}^{\prime \prime}(\cdot, \Delta)$ on $\left[D^{\circ}\left(\tilde{K}_{1}, Y\right), d_{1}^{\circ}\right]$ is measurable with respect to $\left[\mathcal{D}^{\circ}\left(\tilde{K}_{1}, Y\right), d_{1}^{\circ}\right]$ and $\mathcal{R}$ for fixed $\Delta$ owing to $w_{1}^{\prime}(\cdot, \Delta)$ being upper semicontinuous and $\max _{q} w_{1}\left[\cdot, B^{*}\left(x_{q 1}, \Delta\right)\right]$ being continuous on $\left[D^{\circ}\left(\tilde{K}_{1}, Y\right), d_{1}^{\circ}\right]$. A special example of $D^{\circ}\left(\tilde{K}_{1}, Y\right)$ is $D([0,1], R)$. As $\tilde{K}_{1}$ is one-dimensional, for example, the unit interval $[0,1]$ of real numbers, $w_{1}^{\prime \prime}=w_{1}^{\prime}$. As $D^{\circ}\left(\tilde{K}_{1}, Y\right)=D([0,1], R)$, the function $\max _{q} w_{1}\left[F, B^{*}\left(x_{q 1}, \Delta\right)\right]$ is not required for the development of weak convergence theory. Note that the property in the above definition is associated with separability of the space $D^{\circ}\left(\tilde{K}_{1}, Y\right)$.

Similar to the metric space of interest in Section $2,\left[D^{\circ}\left(\tilde{K}_{1}, Y\right), d_{1}^{\circ}\right]$ is a Polish space as indicated by the following theorem. The long proof of the theorem is delegated to the supplementary materials.

Theorem 3.2. The metric space $\left[D^{\circ}\left(\tilde{K}_{1}, Y\right), d_{1}^{\circ}\right]$ is separable and complete, i.e., a Polish space.

In the following, the theorems concerning both the tightness and weak convergence of a sequence of probability measures of interest are the counterparts of the ones in Section 2. First, the theorem below gives the sufficient conditions for the tightness of the sequence of probability measures. Let $\tilde{K}_{1 c}^{*}$ be a countable dense set of $\tilde{K}_{1}$ and contains a countable dense set of the equivalence class [1].

Theorem 3.3. If $\left\{P_{n} \pi_{x_{i}}^{-1}\right\}$ is relatively compact for each $x_{i} \in \tilde{K}_{1 c}^{*}$ and for every positive $\epsilon_{1}$ and $\epsilon_{2}$ there exist $a \Delta$ and an $n_{0}$ such that

$$
P_{n}\left(\left\{F: w_{1}^{\prime \prime}(F, \Delta) \geq \epsilon_{1}\right\}\right) \leq \epsilon_{2}, n \geq n_{0}
$$

then the sequence of probability measures $\left\{P_{n}\right\}$ on $\left[\mathcal{D}^{\circ}\left(\tilde{K}_{1}, Y\right), d_{1}^{\circ}\right]$ is tight.

The next theorem gives the sufficient conditions for weak convergence of the sequence of probability measures. Let $\tilde{K}_{1 P}^{*}$ be a set of $x$ such that $\pi_{x}$ on either $\left[D^{\circ}\left(\tilde{K}_{1}, Y\right), d_{1}^{*}\right]$ or $\left[D^{\circ}\left(\tilde{K}_{1}, Y\right), d_{1}^{\circ}\right]$ is continuous except the set of points of $P$-measure 0 . 
Theorem 3.4. If $\left\{P_{n}\right\}$ is tight and weak convergence of the finite dimensional distributions of $\left\{P_{n}\right\}$ to a probability measure $P$ in $\tilde{K}_{1 P}^{*}$ holds, then the sequence of probability measures $\left\{P_{n}\right\}$ on $\left[\mathcal{D}^{\circ}\left(\tilde{K}_{1}, Y\right), d_{1}^{*}\right]$ or $\left[\mathcal{D}^{\circ}\left(\tilde{K}_{1}, Y\right), d_{1}^{\circ}\right]$ converges weakly to $P$.

\section{Proofs of Main Theorems in Section 2}

For succinctness, the argument $w \in \Omega$ corresponding to a random variable has been suppressed, for example, $\mathcal{F}(x, w)$ being replaced with $\mathcal{F}(x)$.

\subsection{Theorem 2.1}

\subsubsection{Lemmas used for proving Theorem 2.1}

The theorem which establishes the sufficient conditions for the tightness of the sequence of probability measures $\left\{P_{n}\right\}$ on $[\mathcal{C}(K, Y), \rho]$ is based on the following two lemmas. The first lemma gives the sufficient and necessary conditions for the characterization of a relatively compact set in $[C(K, Y), \rho]$.

Lemma 4.1. A set $A$ in $C(K, Y)$ is relatively compact if and only if the following conditions hold:

(i) The set $A_{i}=\left\{\pi_{x_{i}}(F): F \in A\right\}$ is relatively compact for each $x_{i} \in K^{*}$.

$$
\lim _{\Delta \rightarrow 0} \sup _{F \in A} w(F, \Delta)=0
$$

Proof. Since $A$ is relatively compact, it is totally bounded. Thus, for $\epsilon>0$, there exists a finite $\epsilon$-net $\left\{F_{j, \epsilon}, j=1, \ldots, n\right\}$ for $A$ such that $\rho\left(F_{j, \epsilon}, F\right)<\epsilon$ for any $F$ in $A$ and some $j$. Then, $\left\{\pi_{x_{i}}\left(F_{j, \epsilon}\right), j=1, \ldots, n\right\}$ is an $\epsilon$-net for $A_{i}$ because

$$
\left\|\pi_{x_{i}}\left(F_{j, \epsilon}\right)-\pi_{x_{i}}(F)\right\|_{Y} \leq \rho\left(F_{j, \epsilon}, F\right)<\epsilon
$$

Therefore, $A_{i}$ is totally bounded in $Y$. Since $Y$ is complete, $A_{i}$ is relatively compact thus. To prove that condition (ii) holds, let $\left\{F_{j, \epsilon / 3}, j=1, \ldots, m\right\}$ be a finite $\epsilon / 3$-net, i.e., $\rho\left(F_{j, \epsilon / 3}, F\right)<\epsilon / 3$ for any $F$ in $A$ and some $j$. Since $K$ is compact, every $F_{j, \epsilon / 3}$ in the net is uniformly continuous on $K$ and hence 
there exists a $\Delta$ such that $\left\|F_{j, \epsilon / 3}(x)-F_{j, \epsilon / 3}\left(x^{*}\right)\right\|_{Y}<\epsilon / 3$ for any $F_{j, \epsilon / 3}$ in the net and for $\left\|x-x^{*}\right\|_{X}<\Delta$. Then,

$$
\left\|F(x)-F\left(x^{*}\right)\right\|_{Y} \leq 2 \rho\left(F, F_{j, \epsilon / 3}\right)+\left\|F_{j, \epsilon / 3}(x)-F_{j, \epsilon / 3}\left(x^{*}\right)\right\|_{Y}<\epsilon
$$

This gives that $A$ is uniformly equicontinuous and condition (ii) holds.

Conversely, by condition (ii), given $\epsilon>0$, there exists a $\Delta$ such that $w(F, \Delta)<\epsilon / 3$ for all $F$ in $A$. Further, choose in $K^{*}$ a finite $\Delta$-net $\left\{x_{1}, \ldots, x_{k}\right\}$ for $K$ and hence there exists a $x_{i}$ such that $\left\|x-x_{i}\right\|_{X}<\Delta$ and $\| F_{n}(x)-$ $F_{n}\left(x_{i}\right) \|_{Y}<\epsilon / 3$ for any $x$ in $K$ and any sequence $\left\{F_{n}\right\}$ in $A$. Next, by condition (i), there exists a subsequence $\left\{F_{n}^{*}\right\}$ of $\left\{F_{n}\right\}$ such that $\left\{F_{n}^{*}(x)\right\}$ converges to a limit for any $x$ in $K^{*}$. Thus, there exists an $N$ such that for $n, m>N,\left\|F_{n}^{*}\left(x_{i}\right)-F_{m}^{*}\left(x_{i}\right)\right\|_{Y}<\epsilon / 3$ for all $x_{i}$. Then, for any $x$ in $K$,

$$
\begin{aligned}
& \left\|F_{n}^{*}(x)-F_{m}^{*}(x)\right\|_{Y} \\
& \quad \leq\left\|F_{n}^{*}(x)-F_{n}^{*}\left(x_{i}\right)\right\|_{Y}+\left\|F_{n}^{*}\left(x_{i}\right)-F_{m}^{*}\left(x_{i}\right)\right\|_{Y}+\left\|F_{m}^{*}\left(x_{i}\right)-F_{m}^{*}(x)\right\|_{Y} \\
& \quad<\epsilon,
\end{aligned}
$$

and hence $\rho\left(F_{n}^{*}, F_{m}^{*}\right)<\epsilon . \quad\left\{F_{n}^{*}\right\}$ is a Cauchy sequence in the Polish space $[C(K, Y), \rho]$ and this gives that $A$ is relatively compact.

Remark 4.1. The sufficient and necessary conditions for a set of continuous functions to be totally bounded on a compact metric space are uniform boundedness and uniform equicontinuity (see [4], Theorem 7.2; [6], Theorem 2.4.7). Condition (ii) in the above lemma results in the uniform equicontinuity over $A$, while condition (i) corresponds to the one in a more general version of Ascoli's theorem (see [12], Theorem 47.1).

The following lemma establishes the condition for the existence of a sequence of probability measures $P_{n}$ of which values as close to 1 as possible on the set $A$ satisfying condition (i) in the above lemma.

Lemma 4.2. If the sequence of probability measures $\left\{P_{n}\right\}$ on $[\mathcal{C}(K, Y), \rho]$ satisfies that $\left\{P_{n} \pi_{x_{i}}^{-1}\right\}$ is relatively compact for each $x_{i} \in K^{*}$, there exists a measurable set $A$ such that the set $A_{i}=\left\{\pi_{x_{i}}(F): F \in A\right\}$ is relatively compact for each $x_{i} \in K^{*}$ and $P_{n}(A) \geq 1-\epsilon$ for every $\epsilon>0$ and each $n$. 
Proof. Because $Y$ is complete and separable and the sequence of probability measures $\left\{P_{n} \pi_{x_{i}}^{-1}\right\}$ is relatively compact, $\left\{P_{n} \pi_{x_{i}}^{-1}\right\}$ is tight by Prohorov's theorem (see [4], Section 5) and there exists a compact set $S_{i} \subset Y$ such that $P_{n} \pi_{x_{i}}^{-1}\left(S_{i}\right) \geq 1-\epsilon / 2^{i}, i=1,2, \ldots$, for each $n$ and any $\epsilon>0$. Let the measurable set $A=\cap_{i} \pi_{x_{i}}^{-1}\left(S_{i}\right)$. Then,

$$
\begin{aligned}
P_{n}(A) & =1-P_{n}\left\{\cup_{i}\left[\pi_{x_{i}}^{-1}\left(S_{i}\right)\right]^{c}\right\} \\
& \geq 1-\sum_{i} P_{n}\left\{\left[\pi_{x_{i}}^{-1}\left(S_{i}\right)\right]^{c}\right\} \\
& \geq 1-\epsilon .
\end{aligned}
$$

Finally, $A_{i}=\left\{\pi_{x_{i}}(F): F \in A\right\} \subset S_{i}$ and hence the closure of $A_{i}$ is compact, i.e., $A_{i}$ being relatively compact.

\subsubsection{Proof for Theorem 2.1}

By Lemma 4.2, there exists a measurable set $A$ satisfying that the set $A_{i}=\left\{\pi_{x_{i}}(F): F \in A\right\}$ for each $x_{i}$ in $K^{*}$ and $i=1,2, \ldots$, is relatively compact and $P_{n}(A) \geq 1-\epsilon / 2$ for all $n$ and every $\epsilon>0$. By the inequality for $P_{n}$, choose $\Delta_{1 k}$ so that $B_{1 k}=\left\{F: w\left(F, \Delta_{1 k}\right)<1 / k\right\}, k=1, \ldots$, and $P_{n}\left(B_{1 k}\right) \geq 1-\epsilon / 2^{k+1}$ for $n \geq n_{0}$, where $n_{0}$ depends on $B_{1 k}$. Since $[C(K, Y), \rho]$ is Polish, any probability measure defined on $[\mathcal{C}(K, Y), \rho]$ is tight. Thus, there exists a compact set $B_{2 k}$ such that $P_{n}\left(B_{2 k}\right) \geq 1-\epsilon / 2^{k+1}$ for $n<n_{0}$. Further, by Lemma 4.1, there exists a $\Delta_{2 k}$ such that $B_{2 k} \subset$ $\left\{F: w\left(F, \Delta_{2 k}\right)<1 / k\right\}$. Therefore, $P_{n}\left(B_{k}\right) \geq 1-\epsilon / 2^{k+1}$ for all $n$, where $B_{k}=\left\{F: w\left(F, \Delta_{k}\right)<1 / k\right\}, k=1, \ldots$, and $\Delta_{k}=\min \left(\Delta_{1 k}, \Delta_{2 k}\right)$. The set $A \cap\left(\cap_{k} B_{k}\right)$ satisfies conditions (i) and (ii) given in Lemma 4.1, i.e., the set being relatively compact. Let the compact set $K_{1}$ be the closure of $A \cap\left(\cap_{k} B_{k}\right)$. Then,

$$
\begin{aligned}
P_{n}\left(K_{1}\right) & \geq 1-P_{n}\left[A^{c} \cup\left(\cap_{k} B_{k}\right)^{c}\right] \\
& \geq 1-\left[P_{n}\left(A^{c}\right)+\sum_{k=1}^{\infty} P_{n}\left(B_{k}^{c}\right)\right] \\
& \geq 1-\epsilon .
\end{aligned}
$$

for all $n$. 


\subsection{Theorem 2.2}

\subsubsection{Lemma used for proving Theorem 2.2}

Because the space $[C(K, Y), \rho]$ is separable, the ball $\sigma$-field generated by the open balls and the Borel $\sigma$-field are identical. The following lemma indicates that the cylinder $\sigma$-field $\sigma\left(\pi_{x}: x \in K\right.$ ) (see [9], p. 16), i.e., the $\sigma$-field generated by the class of sets $\left\{\pi_{x}^{-1}(S): S \in \mathcal{Y}, x \in K\right\}$, is the Borel $\sigma$-field of subsets of $[C(K, Y), \rho]$. In addition, this lemma also indicates that the class of finite-dimensional sets $\left\{\pi_{x_{1} \cdots x_{k}}^{-1}(S): S \in \mathcal{Y}^{k}\right\}$ is a separating class (see [4], p. 9) for the Borel $\sigma$-field $[\mathcal{C}(K, Y), \rho]$.

Lemma 4.3. $\sigma\left(\pi_{x}: x \in K\right)=[\mathcal{C}(K, Y), \rho]$ and the class of finite-dimensional sets $\left\{\pi_{x_{1} \cdots x_{k}}^{-1}(S): S \in \mathcal{Y}^{k}\right\}$ is a separating class of $[\mathcal{C}(K, Y), \rho]$, where $x_{1}, \ldots, x_{k} \in K$.

Proof. $\pi_{x}$ is Borel, i.e., for each $B$ in $\mathcal{Y}, \pi_{x}^{-1}(B)=\{F: F(x) \in B\} \in$ $[\mathcal{C}(K, Y), \rho]$. Therefore, $\sigma\left(\pi_{x}: x \in K\right) \subset[\mathcal{C}(K, Y), \rho]$.

On the other hand, for any $F_{0}$ in $C(K, Y)$ and $\epsilon>0$,

$$
\bar{B}\left(F_{0}, \epsilon\right)=\left\{F: \rho\left(F, F_{0}\right) \leq \epsilon\right\}=\cap_{x \in K^{*}}\left\{F: F(x) \in \bar{B}\left[F_{0}(x), \epsilon\right]\right\} .
$$

This implies that the closed ball in $[\mathcal{C}(K, Y), \rho]$ falls in the cylinder $\sigma$-field $\sigma\left(\pi_{x}: x \in K\right)$ and the ball $\sigma$-field is a subset of $\sigma\left(\pi_{x}: x \in K\right)$ then. Because $[C(K, Y), \rho]$ is separable, the Borel $\sigma$-field $[\mathcal{C}(K, Y), \rho]$ is the ball $\sigma$-field and also a subset of $\sigma\left(\pi_{x}: x \in K\right)$ thus, i.e., $[\mathcal{C}(K, Y), \rho] \subset \sigma\left(\pi_{x}: x \in K\right)$. Therefore, $\sigma\left(\pi_{x}: x \in K\right)=[\mathcal{C}(K, Y), \rho]$.

$\left\{\pi_{x_{1} \cdots x_{k}}^{-1}(S): S \in \mathcal{Y}^{k}\right\} \subset[\mathcal{C}(K, Y), \rho]$ because $\pi_{x_{1} \cdots x_{k}}$ is continuous and hence measurable with respect to $[\mathcal{C}(K, Y), \rho]$ and $\mathcal{Y}^{k}$. Further, because the class of finite-dimensional sets $\left\{\pi_{x_{1} \cdots x_{k}}^{-1}(S): S \in \mathcal{Y}^{k}\right\}$ is a $\pi$ system and generates the cylinder $\sigma$-field, it is a separating class owing to the Borel $\sigma$-field $[\mathcal{C}(K, Y), \rho]$ being the cylinder $\sigma$-field $\sigma\left(\pi_{x}: x \in K\right)$.

\subsubsection{Proof of Theorem 2.2}

Since $\left\{P_{n}\right\}$ is tight, it is relatively compact by Prohorov's theorem. Thus, there exists a further subsequence $\left\{P_{n_{i(m)}}\right\}$ of every subsequence $\left\{P_{n_{i}}\right\}$ of $\left\{P_{n}\right\}$ converges weakly to a probability measure $P$. Then, $\left\{P_{n_{i(m)}} \pi_{x_{1} \cdots x_{k}}^{-1}\right\}$ converges weakly to $P \pi_{x_{1} \cdots x_{k}}^{-1}$ by the mapping theorem and hence $P \pi_{x_{1} \cdots x_{k}}^{-1}=$ 
$Q_{x_{1} \cdots x_{k}}$. Since the class of finite-dimensional sets is a separating class of $[\mathcal{C}(K, Y), \rho]$ by Lemma 4.3 , the result follows by Theorem 2.6 of [4].

\subsection{Theorem 2.4 (a)}

$\left\{\mathcal{F}_{n}\right\}$ satisfying the conditions given in Theorem 2.3 is proved. The following lemma indicates that $\left\{\mathcal{F}_{n}\right\}$ satisfies the equation

$$
\lim _{\Delta \rightarrow 0} \limsup _{n \rightarrow \infty} P\left[w\left(\mathcal{F}_{n}, \Delta\right) \geq \epsilon\right]=0,
$$

given in Theorem 2.3.

\subsubsection{Lemma used for proving Theorem 2.4 (a)}

In the following lemma, $Y$ is a real separable Banach space, not necessarily a cotype 2 Banach space.

Lemma 4.4. The sequence of $C(K, Y)$-valued random variables $\left\{\mathcal{F}_{n}\right\}$ satisfies the condition

$$
\lim _{\Delta \rightarrow 0} \limsup _{n \rightarrow \infty} P\left[w\left(\mathcal{F}_{n}, \Delta\right) \geq \epsilon\right]=0,
$$

given in Theorem 2.3 .

Proof. The following inequality is proved first,

$$
P\left[w\left(\mathcal{F}_{n}, \Delta\right) \geq 2 \epsilon_{1}\right] \leq \sum_{i=1}^{v} P\left[\sup _{s \in B\left(x_{i}, 2 \Delta\right)}\left\|\mathcal{F}_{n}(s)-\mathcal{F}_{n}\left(x_{i}\right)\right\|_{Y} \geq \epsilon_{1}\right],
$$

for given $\epsilon_{1}>0$, where $0 \leq\left\|x_{1}\right\|_{X} \leq \cdots \leq\left\|x_{v}\right\|_{X} \leq 1$ and $\left\{x_{i}: i=1, \ldots, v\right\}$ is a finite $\Delta$-net for $K$. As $\|s-x\|_{X} \leq \Delta, s$ and $x$ fall in some neighborhood $B\left(x_{i}, 2 \Delta\right)$ because there exists a $x_{i}$ such that $\left\|x-x_{i}\right\|_{X}<\Delta$ and hence

$$
\left\|s-x_{i}\right\|_{X} \leq\|s-x\|_{X}+\left\|x-x_{i}\right\|_{X}<2 \Delta .
$$

Then,

$$
\|F(s)-F(x)\|_{Y} \leq\left\|F(s)-F\left(x_{i}\right)\right\|_{Y}+\left\|F\left(x_{i}\right)-F(x)\right\|_{Y}
$$

and hence 


$$
w(F, \Delta) \leq 2 \max _{1 \leq i \leq v} \sup _{s \in B\left(x_{i}, 2 \Delta\right)}\left\|F(s)-F\left(x_{i}\right)\right\|_{Y}
$$

The objective inequality is obtained by

$$
\begin{aligned}
P\left[w\left(\mathcal{F}_{n}, \Delta\right) \geq 2 \epsilon_{1}\right] & \leq P\left[\max _{1 \leq i \leq v} \sup _{s \in B\left(x_{i}, 2 \Delta\right)}\left\|\mathcal{F}_{n}(s)-\mathcal{F}_{n}\left(x_{i}\right)\right\|_{Y} \geq \epsilon_{1}\right] \\
& \leq \sum_{i=1}^{v} P\left[\sup _{s \in B\left(x_{i}, 2 \Delta\right)}\left\|\mathcal{F}_{n}(s)-\mathcal{F}_{n}\left(x_{i}\right)\right\|_{Y} \geq \epsilon_{1}\right]
\end{aligned}
$$

Based on the above inequality, the next step is to prove the inequality

$$
\begin{aligned}
& P\left[w\left(\mathcal{F}_{n}, \Delta\right) \geq 2 \epsilon_{1}\right] \\
& \leq v\left[6 P\left(\max _{k \leq m}\left\|S_{k}\right\|_{Y} \geq \frac{n^{1 / 2} \epsilon_{1}}{3}\right)+P\left(\|\xi\|_{Y} \geq \frac{n^{1 / 2} \epsilon_{1}}{3}\right)\right],
\end{aligned}
$$

where $S_{k}=\sum_{i=1}^{k} \xi_{i}$ and $m=\lfloor 2 n L \Delta\rfloor$. Because

$$
\begin{aligned}
& \mathcal{F}_{n}(s)-\mathcal{F}_{n}\left(x_{i}\right) \\
& =\frac{\left(S_{\lfloor n \phi(s)\rfloor}-S_{\left\lfloor n \phi\left(x_{i}\right)\right\rfloor}\right)}{n^{1 / 2}}+\frac{c_{n}(s) \xi_{\lfloor n \phi(s)\rfloor+1}}{n^{1 / 2}}-\frac{c_{n}\left(x_{i}\right) \xi_{\left\lfloor n \phi\left(x_{i}\right)\right\rfloor+1}}{n^{1 / 2}}
\end{aligned}
$$

and by the Lipschitz condition imposed on $\phi$,

$$
\left|n \phi(s)-n \phi\left(x_{i}\right)\right| \leq n L\left\|s-x_{i}\right\|_{X}<2 n L \Delta,
$$

the set in the sample space satisfies

$$
\begin{aligned}
&\left\{\sup _{s \in B\left(x_{i}, 2 \Delta\right)}\left\|\mathcal{F}_{n}(s)-\mathcal{F}_{n}\left(x_{i}\right)\right\|_{Y} \geq \epsilon_{1}\right\} \\
& \subset\left\{\left\{\sup _{s \in B\left(x_{i}, 2 \Delta\right)}\left\|\frac{\left(S_{\lfloor n \phi(s)\rfloor}-S_{\left\lfloor n \phi\left(x_{i}\right)\right\rfloor}\right)}{n^{1 / 2}}\right\|_{Y} \geq \frac{\epsilon_{1}}{3}\right\}\right. \\
&\left.\cup\left\{\sup _{s \in B\left(x_{i}, 2 \Delta\right), s \neq x_{i}} \frac{\left\|\xi_{j_{1}(s)+1}\right\|_{Y}}{n^{1 / 2}} \geq \frac{\epsilon_{1}}{3}\right\} \cup\left\{\frac{\left\|\xi_{j_{2}+1}\right\|_{Y}}{n^{1 / 2}} \geq \frac{\epsilon_{1}}{3}\right\}\right\} \\
& \subset\left\{\left\{\max _{\max \left(0, j_{2}-m\right)<k \leq j_{2}}\left\|\frac{S_{k}-S_{j_{2}}}{n^{1 / 2}}\right\|_{Y} \geq \frac{\epsilon_{1}}{3}\right\}\right. \\
& \cup\left\{\max _{j_{2}<k \leq\left(j_{2}+m\right)}\left\|\frac{S_{k}-S_{j_{2}}}{n^{1 / 2}}\right\|_{Y} \geq \frac{\epsilon_{1}}{3}\right\}
\end{aligned}
$$




$$
\begin{aligned}
& \cup\left\{\max _{\max \left(0, j_{2}+1-m\right) \leq k<j_{2}+1} \frac{\left\|\xi_{k}\right\|_{Y}}{n^{1 / 2}} \geq \frac{\epsilon_{1}}{3}\right\} \\
& \left.\cup\left\{\max _{j_{2}+1<k \leq\left(j_{2}+1+m\right)} \frac{\left\|\xi_{k}\right\|_{Y}}{n^{1 / 2}} \geq \frac{\epsilon_{1}}{3}\right\} \cup\left\{\frac{\left\|\xi_{j_{2}+1}\right\|_{Y}}{n^{1 / 2}} \geq \frac{\epsilon_{1}}{3}\right\}\right\},
\end{aligned}
$$

where $j_{1}(s)=\lfloor n \phi(s)\rfloor$ and $j_{2}=\left\lfloor n \phi\left(x_{i}\right)\right\rfloor$. By Proposition 2.3 of [10] and subadditivity, the inequality holds.

To complete the proof, the Etemadi's inequality (see [4], M19) can be generalized for the $Y$-valued random variable, i.e.,

$$
P\left(\max _{k \leq m}\left\|S_{k}\right\|_{Y} \geq 3 \epsilon_{1}\right) \leq 3 \max _{k \leq m} P\left(\left\|S_{k}\right\|_{Y} \geq \epsilon_{1}\right) .
$$

Then, by the Etemadi's inequality, Lemma 3.1 of [10], and the condition for the size of the net for $K$,

$$
\begin{aligned}
P\left[w\left(\mathcal{F}_{n}, \Delta\right) \geq 2 \epsilon_{1}\right] & \leq v\left[6 P\left(\max _{k \leq m}\left\|S_{k}\right\|_{Y} \geq \frac{n^{1 / 2} \epsilon_{1}}{3}\right)+P\left(\|\xi\|_{Y} \geq \frac{n^{1 / 2} \epsilon_{1}}{3}\right)\right] \\
& \leq \frac{42 L n h\left(\Delta^{-1}\right)}{m} \max _{k \leq m} P\left(\left\|S_{k}\right\|_{Y} \geq \frac{n^{1 / 2} \epsilon_{1}}{9}\right) \\
& \leq \frac{3402 L \lambda^{2} h\left(\Delta^{-1}\right)}{\epsilon_{1}^{2}} \max _{k \leq m} P\left(\left\|S_{k}\right\|_{Y} \geq m^{1 / 2} \lambda\right) \\
& =\frac{3402 L \lambda^{2} h\left(\Delta^{-1}\right)}{\epsilon_{1}^{2}} \max _{k \leq m} P\left(\left\|k^{-1 / 2} S_{k}\right\|_{Y} \geq m^{1 / 2} k^{-1 / 2} \lambda\right) \\
& \leq \frac{3402 L \lambda^{2} h\left(\frac{162 L \lambda^{2}}{\epsilon_{1}^{2}}\right) \exp \left\{\frac{-[\lambda-M(\xi)-1]^{2}}{2[\Sigma(\xi)]^{2}}\right\}}{\epsilon_{1}^{2}}
\end{aligned}
$$

for $\Delta<\delta$, where $M(\xi)$ is the median of $\|\xi\|_{Y}, k^{-1 / 2} S_{k}$ is centered Radon Gaussian owing to $\xi$ being centered Radon Gaussian and $\lambda=9^{-1} m^{-1 / 2} n^{1 / 2} \epsilon_{1}$. By the condition for the size of the net for $K$ again, given $\epsilon_{1}$ and $\epsilon_{2}$, there exist a $\Delta_{0}$ associated with a $\lambda_{0}$ and an $n_{0}$ such that

$$
P\left[w\left(\mathcal{F}_{n}, \Delta_{0}\right) \geq 2 \epsilon_{1}\right] \leq \epsilon_{2}, n \geq n_{0}
$$

\subsubsection{Proof of Theorem 2.4 (a)}

By Lemma 4.4, the equation in Theorem 2.3 holds. The convergence 
of the finite-dimensional distributions of $\mathcal{F}_{n}$ to those of the random variable having a generalized Wiener measure given in Definition 2.1 as its distribution is proved as follows.

If $x=0,\left\{\mathcal{F}_{n}(0)=0\right\}$ converges in distribution to $\mathcal{W}_{\phi}(0)$. If $x \neq 0$ and $c_{n}(x)=0, \mathcal{F}_{n}(x)$ is a centered Radon Gaussian variable with $\Sigma\left[\mathcal{F}_{n}(x)\right]=$ $(\lfloor n \phi(x)\rfloor / n)^{1 / 2}$. Then, $\left\{\mathcal{F}_{n}(x)\right\}$ converges in distribution to a centered Radon Gaussian variable $\mathcal{W}_{\phi}(x)$ with $\Sigma\left[\mathcal{W}_{\phi}(x)\right]=[\phi(x)]^{1 / 2}$ owing to $Y$ being a cotype 2 Banach space and by employing Theorem 10.7 of [10] first and then the converging together lemma. If $x \neq 0$ and $c_{n}(x) \neq 0$, $\mathcal{F}_{n}(x)=S_{\lfloor\phi(x)\rfloor} / n^{1 / 2}+\xi_{n}^{*}(x)$, where $\xi_{n}^{*}(x)=c_{n}(x) \xi_{\lfloor n \phi(x)\rfloor+1} / n^{1 / 2}$. By Lemma 3.1 of [10], for any $\epsilon_{1}>0$ and $\epsilon_{2}>0$, there exists an $n_{0}$ such that for $n>n_{0}$

$$
\begin{aligned}
P\left(\left\|\xi_{n}^{*}(x)\right\|_{Y}>\epsilon_{1}\right) & \leq P\left[\left|\|\xi\|_{Y}-M(\xi)\right|>\frac{n^{1 / 2} \epsilon_{1}}{c_{n}(x)}-M(\xi)\right] \\
& \leq \exp \left\{\frac{-\left[\frac{n^{1 / 2} \epsilon_{1}}{c_{n}(x)}-M(\xi)\right]^{2}}{2[\Sigma(\xi)]^{2}}\right\} \\
& <\epsilon_{2} .
\end{aligned}
$$

Because $\left\{S_{\lfloor\phi(x)\rfloor} / n^{1 / 2}\right\}$ converges in distribution to a centered Radon Gaussian variable $\mathcal{W}_{\phi}(x)$ with $\Sigma\left[\mathcal{W}_{\phi}(x)\right]=[\phi(x)]^{1 / 2},\left\{\mathcal{F}_{n}(x)\right\}$ converges in distribution to the centered Radon Gaussian variable $\mathcal{W}_{\phi}(x)$ by the converging together lemma.

To prove that $\left\{\mathcal{F}_{n}(x)\right\}$ and $\left\{\mathcal{F}_{n}(y)-\mathcal{F}_{n}(x)\right\}$ converge in distribution to $\mathcal{W}_{\phi}(x)$ and $\mathcal{W}_{\phi}(y)-\mathcal{W}_{\phi}(x)$, respectively, let $\phi(y) \geq \phi(x)$, where $\mathcal{W}_{\phi}(x)$ and $\mathcal{W}_{\phi}(y)$ are centered Radon Gaussian variables and $\mathcal{W}_{\phi}(y)-\mathcal{W}_{\phi}(x)$ is independent of $\mathcal{W}_{\phi}(x)$. Since both $S_{\lfloor n \phi(y)\rfloor} / n^{1 / 2}$ and $S_{\lfloor n \phi(x)\rfloor} / n^{1 / 2}$ are centered Radon Gaussian variables, $\left(S_{\lfloor n \phi(y)\rfloor}-S_{\lfloor n \phi(x)\rfloor}\right) / n^{1 / 2}$ independent of $S_{\lfloor n \phi(x)\rfloor} / n^{1 / 2}$, and $\left\{c_{n}(y) \xi_{\lfloor n \phi(y)\rfloor+1} / n^{1 / 2}\right\}$ and $\left\{c_{n}(x) \xi_{\lfloor n \phi(x)\rfloor+1} / n^{1 / 2}\right\}$ converge to 0 in probability as $n \rightarrow \infty,\left\{\left[\mathcal{F}_{n}(x), \mathcal{F}_{n}(y)-\mathcal{F}_{n}(x)\right]\right\}$ converges in distribution to $\left[\mathcal{W}_{\phi}(x), \mathcal{W}_{\phi}(y)-\mathcal{W}_{\phi}(x)\right]$ by the mapping theorem and the converging together lemma. By employing the mapping theorem again, $\left\{\left[\mathcal{F}_{n}(x), \mathcal{F}_{n}(y)\right]\right\}$ converges in distribution to $\left[\mathcal{W}_{\phi}(x), \mathcal{W}_{\phi}(y)\right]$. By the analogous argument, it can be proved that $\left\{\left[\mathcal{F}_{n}\left(x_{1}\right), \ldots, \mathcal{F}_{n}\left(x_{k}\right)\right]\right\}$ converges in distribution to $\left[\mathcal{W}_{\phi}\left(x_{1}\right), \ldots, \mathcal{W}_{\phi}\left(x_{k}\right)\right]$, where $\phi\left(x_{1}\right) \leq \cdots \leq \phi\left(x_{k}\right)$ and $\mathcal{W}_{\phi}\left(x_{1}\right), \mathcal{W}_{\phi}\left(x_{2}\right)-\mathcal{W}_{\phi}\left(x_{1}\right), \ldots, \mathcal{W}_{\phi}\left(x_{k}\right)-\mathcal{W}_{\phi}\left(x_{k-1}\right)$ are independent. Thus, 
by Theorem 2.3, $\left\{\mathcal{F}_{n}\right\}$ converges in distribution to the random variable $\mathcal{W}_{\phi}$ and the distribution of $\mathcal{W}_{\phi}$ is the generalized Wiener measure $W_{\phi}$.

\section{Proofs of Main Theorems in Section 3}

\subsection{Basic lemmas in Section 3}

The following three lemmas are useful for the proofs of the main theorems in Section 3. The first lemma indicates that the operators in $D\left(\tilde{K}_{1}, Y\right)$ can not "jump" uncountably along every path $[0,1]_{x}=\left\{c x /\|x\|_{X}: 0 \leq c \leq\right.$ $1\}$.

Lemma 5.1. For each $F$ in $D\left(\tilde{K}_{1}, Y\right)$,

$$
\lim _{\Delta \rightarrow 0} w_{1}^{\prime}(F, \Delta, x)=0
$$

for any $x \in \tilde{K}$. Further, if

$$
\lim _{\Delta \rightarrow 0} w_{1}^{\prime}(F, \Delta)=0
$$

the following equation

$$
\lim _{\Delta \rightarrow 0} \sup _{x \in \tilde{K}_{1}} w_{1}^{\prime}(F, \Delta, x)=0
$$

holds.

Proof. By the proof analogous to Lemma 1 of Section 12 of [4] and the arguments in the section, $\lim _{\Delta_{n} \rightarrow 0} w_{1}^{\prime}\left(F, \Delta_{n}, x\right)=0$ for any $x$ in $\tilde{K}_{1}$, where $\Delta_{n} \rightarrow 0$ as $n \rightarrow \infty$.

Next is to prove that the equation $\lim _{\Delta \rightarrow 0} w_{1}^{\prime}(F, \Delta)=0$ implies the equation $\lim _{\Delta \rightarrow 0} \sup _{x \in \tilde{K}_{1}} w_{1}^{\prime}(F, \Delta, x)=0$. Because $w_{1}^{\prime}(F, \Delta, x) \leq \max _{i}$ $w_{1}\left\{F,\left[s_{i-1}, s_{i}\right)_{x}\right\}$,

$$
\sup _{x \in \tilde{K}_{1}} w_{1}^{\prime}(F, \Delta, x) \leq \sup _{x \in \tilde{K}_{1}} \max _{i} w_{1}\left\{F,\left[s_{i-1}, s_{i}\right)_{x}\right\} \leq \max _{i} w_{1}\left\{F,\left[s_{i-1}, s_{i}\right)\right\},
$$

for any $\Delta$-sparse division $\left\{s_{i}\right\}$. The result holds thus. 
Remark 5.1. The first two equations in Lemma 5.1 indicate the difference between $D\left(\tilde{K}_{1}, Y\right)$ and $D([0,1], R)$ (see [4], Lemma 1, p. 122), i.e., the first equation satisfied in $D\left(\tilde{K}_{1}, Y\right)$ and the second equation satisfied in $D([0,1], R)$. Because

$$
w_{1}^{\prime}(F, \Delta)=\inf _{\left\{s_{i}\right\}} \max _{i} \sup _{x \in \tilde{K}_{1}} w_{1}\left\{F,\left[s_{i-1}, s_{i}\right)_{x}\right\},
$$

it implies that the division $\left\{s_{i}\right\}$ is path independent, i.e., not relying on the separate path $[0,1]_{x}$. In addition, the first equation implies that there are only countably many discontinuities along every path $[0,1]_{x}$. On the other hand, the second equation indicates that there are only countably many discontinuous equivalence classes. As $X$ is one-dimensional, the two equations are equivalent. In addition, the cadlag operator in $D\left(\tilde{K}_{1}, Y\right)$ satisfying the third equation also satisfies the first equation.

The second lemma indicates that $\left[D\left(\tilde{K}_{1}, Y\right), d_{1}\right]$ is a metric space. Since the proof is quite routine and not presented.

Lemma 5.2. $d_{1}$ is a metric on $D\left(\tilde{K}_{1}, Y\right)$ and hence $\left[D\left(\tilde{K}_{1}, Y\right), d_{1}\right]$ is a metric space.

The third lemma indicates the measurability of the projection function $\pi_{x_{1} \ldots x_{k}}$.

Lemma 5.3. $\pi_{x_{1} \ldots x_{k}}:\left[D\left(\tilde{K}_{1}, Y\right), d_{1}\right] \rightarrow Y^{k}$ is measurable with respect to the $\sigma$-fields $\left[\mathcal{D}\left(\tilde{K}_{1}, Y\right), d_{1}\right]$ and $\mathcal{Y}^{k}$, i.e., a Borel measurable function.

Proof. It suffices to prove that $\pi_{x}$ is Borel measurable. Then, by Theorem 4.43 and Lemma 4.48 of [3], $\pi_{x_{1} \ldots x_{k}}$ is Borel measurable. If the point convergence of a sequence of continuous operators $\left\{h_{m}\right\}$ to $\pi_{x}$ can be proved, $\pi_{x}$ is Borel measurable by Corollary 4.25 and Lemma 4.30 of [3], where $h_{m}: D\left(\tilde{K}_{1}, Y\right) \rightarrow Y, m=1,2, \ldots, 0<\|x\|_{X}=c<1$. To establish the sequence of continuous operators $\left\{h_{m}\right\}$, the result that $F_{n} \underset{n \rightarrow \infty}{\longrightarrow} F$ in the Skorohod topology implies the point convergence of $F_{n}$ at the continuity points of $F$ with the restriction to $[0,1]_{x}$ is required. To prove the result, suppose that $\left\{F_{n}\right\}$ converges to $F$ in the Skorohod topology and $x_{0} \in[0,1]_{x}$ is the continuity point of $F$ with restriction to $[0,1]_{x}$. Then, there exists an $n_{0}$ such that $d_{1}\left(F_{n}, F\right)<\epsilon_{1}$ for any positive $\epsilon_{1}$ and $n>n_{0}$. Thus, there 
exists a $\lambda_{n} \in \Lambda_{1}$ such that $\left\|\lambda_{n}-I\right\|_{\text {sup }}<\epsilon_{1}$ and $\left\|F_{n}-F \lambda_{n}\right\|_{\text {sup }}<\epsilon_{1}$. Since $F$ is continuous at $x_{0}$, there exists a $\delta$ such that $\left\|F\left(x^{*}\right)-F\left(x_{0}\right)\right\|_{Y}<\epsilon_{2}$ for $\left\|x^{*}-x_{0}\right\|_{X}<\delta, x^{*} \in[0,1]_{x}$, and any given positive $\epsilon_{2}$. Then, for $\epsilon_{1}<\delta$,

$$
\left\|F_{n}\left(x_{0}\right)-F\left(x_{0}\right)\right\|_{Y} \leq\left\|F_{n}-F \lambda_{n}\right\|_{\text {sup }}+\left\|F\left[\lambda_{n}\left(x_{0}\right)\right]-F\left(x_{0}\right)\right\|_{Y} \leq \epsilon_{1}+\epsilon_{2} .
$$

Next is to prove the point convergence of $\left\{h_{m}\right\}$ to $\pi_{x}$. Let the Bochner integral $h_{m}(F)=m \int_{E_{m}} F^{*} d u$, where $F^{*}: E_{m} \rightarrow Y$ is defined by $F^{*}(t)=$ $F\left(t x /\|x\|_{X}\right), E_{m}=\{t: c \leq t<c+1 / m, t \in R\}$ is the interval of real numbers, and $u$ is the Lebesgue measure on the real line. Note that $\int_{E_{m}}\left\|F^{*}(t)\right\|_{Y} d u(t)<\infty$ (see [3], Theorem 11.43) and hence $F^{*}$ is Bochner integrable, i.e., $h_{m}$ being well-defined. Note that the analogous result to the one in Lemma 5.1 also follows for the domain $[c, c+1 / m)_{x}$, i.e.,

$$
\lim _{\Delta \rightarrow 0} \inf _{\left\{s_{i}\right\}} \max _{i} w_{1}\left\{F,\left[s_{i-1}, s_{i}\right)_{x}\right\}=0,
$$

where $\left\{s_{i}\right\}$ is any $\Delta$-sparse division for the interval $[c, c+1 / m]$ of real numbers. Therefore, there are only countably many discontinuities of $F$ along the path $[c, c+1 / m)_{x}$ and hence only countably many discontinuities of $F^{*}$ in $E_{m}$. As $F_{n} \underset{n \rightarrow \infty}{\longrightarrow} F$ in the Skorohod topology, $F_{n}^{*}(t) \underset{n \rightarrow \infty}{\longrightarrow} F^{*}(t)$ for points $t$ outside a Lebesgue measure 0 and there exist $\lambda_{n} \in \Lambda_{1}$ and some positive integer $N$ such that

$$
\begin{aligned}
\left\|F_{n}\right\|_{\text {sup }} & \leq\left\|F_{n}-F \lambda_{n}\right\|_{\text {sup }}+\left\|F \lambda_{n}\right\|_{\text {sup }} \\
& \leq d_{1}\left(F_{n}, F\right)+\left\|F \lambda_{n}\right\|_{\text {sup }}+\epsilon / 2 \\
& \leq\left\|F \lambda_{n}\right\|_{\text {sup }}+\epsilon \\
& =\|F\|_{\text {sup }}+\epsilon
\end{aligned}
$$

for $n>N$ and any $\epsilon>0$. Therefore, $h_{m}\left(F_{n}\right) \underset{n \rightarrow \infty}{\longrightarrow} h_{m}(F)$ by dominated convergence theorem (see [3], Theorem 11.45) for Bochner integral. Therefore, $h_{m}$ is continuous in the Skorohod topology by Theorem 21.3 of [12] and hence Borel measurable. By the right continuity of $F$ at $x, \| h_{m}(F)-$ $\pi_{x}(F) \|_{Y} \underset{m \rightarrow \infty}{\longrightarrow} 0$. Finally, $\pi_{0}$ is continuous in the Skorohod topology and hence Borel measurable owing to

$$
\left\|\pi_{0}\left(F_{n}\right)-\pi_{0}(F)\right\|_{Y}=\left\|F_{n}(0)-F(0)\right\|_{Y} \leq d_{1}\left(F_{n}, F\right) .
$$


The proof for $\pi_{x},\|x\|_{X}=1$, being continuous is analogous to the one for $\pi_{0}$.

\subsection{Theorem 3.3}

\subsubsection{Lemmas used for proving Theorem 3.3}

The following two lemmas are the counterparts of Lemma 4.1 and Lemma 4.2 in the proof of Theorem 2.1.

Lemma 5.4. $A$ set $A$ in $\left[D^{\circ}\left(\tilde{K}_{1}, Y\right), d_{1}^{\circ}\right]$ is relatively compact if the following conditions hold:

(i) The set $A_{i}=\left\{\pi_{x_{i}}(F): F \in A\right\}$ is relatively compact for each $x_{i} \in \tilde{K}_{1 c}^{*}$.

(ii)

$$
\lim _{\Delta \rightarrow 0} \sup _{F \in A} w_{1}^{\prime \prime}(F, \Delta)=0 .
$$

On the other hand, if the set $A$ is relatively compact, then condition (ii) holds.

Proof. The proof of sufficiency can be divided into two parts. The set $A$ being totally bounded in the sense of $d_{1}^{*}$ is established in the first part which the set $A$ being relatively compact in the sense of $d_{1}^{\circ}$ can be deduced from and established in the second part.

Let the division $0=t_{n 0}<t_{n 1}<\cdots<t_{n k_{n}}=1$ with $\max _{i}\left(t_{n i}-\right.$ $\left.t_{n(i-1)}\right) \leq 1 / n$ and $n=1,2, \ldots$ In addition, let $\hat{F}_{\Delta}(x)=F\left(x_{n i}\right)$ for $x \in$ $\left[t_{n(i-1)}, t_{n i}\right)$ and $\hat{F}_{\Delta}(x)=F\left(x_{q 1}\right)$ for $x \in B^{*}\left(x_{q 1}, \Delta\right)$, where $x_{n i} \in\left[t_{n(i-1)}\right]$ and $\left(\left\{x_{n i}\right\} \cup\left\{x_{q 1}\right\}\right) \subset \tilde{K}_{1 c}^{*}$. By condition (ii), for given $\epsilon>0$, there exist $\Delta$ and $\hat{F}_{\Delta}$ depending on $F$ such that $w_{1}^{\prime}(F, \Delta)<\epsilon, \max _{q} w_{1}\left[F, B^{*}\left(x_{q 1}, \Delta\right)\right]<\epsilon$ and $d_{1}^{*}\left(\hat{F}_{\Delta}, F\right)<\epsilon$ for any $F \in A$. The inequality $d_{1}^{*}\left(\hat{F}_{\Delta}, F\right)<\epsilon$ has been established in the proof of Theorem 3.2 given in the supplementary material. Let $S$ be the closure of the set $\left(\cup_{i}\left\{F\left(x_{n i}\right): F \in A\right\}\right) \cup\left(\cup_{q}\left\{F\left(x_{q 1}\right): F \in A\right\}\right)$. $S$ is compact by condition (i). Thus, there exists a finite $\epsilon$-net $H$ for $S$. Let $\mathcal{N}$ be the finite set of the operators that assume on each $\left[t_{n(i-1)}, t_{n i}\right)$ and $B^{*}\left(x_{q 1}, \Delta\right)$ a constant value from $H$. Then, let $\hat{F}_{\Delta}^{*}$ in $\mathcal{N}$ defined by 
$\hat{F}_{\Delta}^{*}(x)=h_{n i}$ for $x \in\left[t_{n(i-1)}, t_{n i}\right)$ and $\hat{F}_{\Delta}^{*}(x)=h_{q 1}$ for $x \in B^{*}\left(x_{q 1}, \Delta\right)$, where $\left\|h_{n i}-F\left(x_{n i}\right)\right\|_{Y}<\epsilon$ and $\left\|h_{q 1}-F\left(x_{q 1}\right)\right\|_{Y}<\epsilon$. Thus,

$$
\begin{aligned}
d_{1}^{*}\left(\hat{F}_{\Delta}^{*}, F\right) & \leq\left\|\hat{F}_{\Delta}^{*}-\hat{F}_{\Delta}\right\|_{\text {sup }}+d_{1}^{*}\left(\hat{F}_{\Delta}, F\right) \\
& <2 \epsilon .
\end{aligned}
$$

Therefore, $\mathcal{N}$ is a finite $2 \epsilon$-net for $A$ and $A$ is totally bounded in the sense of $d_{1}^{*}$.

Next is to prove that $A$ is totally bounded in the sense of $d_{1}^{\circ}$ and hence $A$ is relatively compact because of completeness of $D^{\circ}\left(\tilde{K}_{1}, Y\right)$ (see [4], M5). The inequality $d_{1}^{\circ}\left(F_{1}, F_{2}\right) \leq 4 \Delta+\sup _{x \in \tilde{K}_{1}} w_{1}^{\prime}\left(F_{1}, \Delta, x\right)$ given that $0<\Delta \leq$ $1 / 4$ and $d_{1}^{*}\left(F_{1}, F_{2}\right)<\Delta^{2}$ has been established in the proof of Lemma 3.1 given in the supplementary materials. Using this inequality, the following inequality

$$
\begin{aligned}
d_{1}^{\circ}\left(F_{1}, F_{2}\right) & \leq 4 \Delta+\sup _{x \in \tilde{K}_{1}} w_{1}^{\prime}\left(F_{1}, \Delta, x\right) \\
& \leq 4 \Delta+w_{1}^{\prime}\left(F_{1}, \Delta\right)
\end{aligned}
$$

can be established if $0<\Delta \leq 1 / 4$ and $d_{1}^{*}\left(F_{1}, F_{2}\right)<\Delta^{2}$. Furthermore, there exists a $\Delta<\min (\epsilon / 8,1 / 4)$ such that $4 \Delta+w_{1}^{\prime}(F, \Delta)<\epsilon$ for any positive $\epsilon$ and any $F \in A$ by condition (ii). Then, the $\Delta^{2}$-net in the sense of the metric $d_{1}^{*}$ is a finite $\epsilon$-net in the sense of the metric $d_{1}^{\circ}$ because for any $F \in A$ there exists an operator $\hat{F}_{\Delta}^{*}$ in the net satisfying $d_{1}^{*}\left(F, \hat{F}_{\Delta}^{*}\right)<\Delta^{2}$ and hence $d_{1}^{\circ}\left(F, \hat{F}_{\Delta}^{*}\right)<\epsilon$. Therefore, $A$ is totally bounded in the sense of $d_{1}^{\circ}$.

The proof of $A$ being relative compact implying condition (ii) is given in the following. By the property given in Definition 3.6, there exists a sequence $\left\{\Delta_{n}\right\}$ such that both sequences $\left\{w_{1}^{\prime}\left(\cdot, \Delta_{n}\right)\right\}$ and $\left\{\max _{q} w_{1}\left[\cdot, B^{*}\left(x_{q 1}, \Delta_{n}\right)\right]\right\}$ defined on $D^{\circ}\left(\tilde{K}_{1}, Y\right)$ satisfying $w_{1}^{\prime}\left(F, \Delta_{n}\right) \downarrow 0$ and $\max _{q} w_{1}\left[F, B^{*}\left(x_{q 1}, \Delta_{n}\right)\right] \downarrow$ 0 for every $F$ in $D^{\circ}\left(\tilde{K}_{1}, Y\right)$. Further, $w_{1}^{\prime}(\cdot, \Delta)$ is upper semi-continuous for fixed $\Delta$ while $\max _{q} w_{1}\left[\cdot, B^{*}\left(x_{q 1}, \Delta\right)\right]$ is continuous for fixed $\Delta$. Thus, by Dini's theorem given in M8 of [4], condition (ii) holds.

Lemma 5.5. If the sequence of probability measures $\left\{P_{n}\right\}$ on the metric space $\left[\mathcal{D}^{\circ}\left(\tilde{K}_{1}, Y\right), d_{1}^{\circ}\right]$ satisfies that $\left\{P_{n} \pi_{x_{i}}^{-1}\right\}$ is relatively compact for each $x_{i} \in \tilde{K}_{1 c}^{*}$, there exists a measurable set $A$ such that $A_{i}=\left\{\pi_{x_{i}}(F): F \in A\right\}$ is relatively compact for each $x_{i} \in \tilde{K}_{1 c}^{*}$ and $P_{n}(A) \geq 1-\epsilon$ for every $\epsilon>0$. 
Proof. The proof is analogous to the one of Lemma 4.2.

\subsubsection{Proof of Theorem 3.3}

By replacing the function $w$ with the function $w_{1}^{\prime \prime}$, using Theorem 3.2 and by using Lemma 5.4 and Lemma 5.5 in place of Lemma 4.1 and Lemma 4.2 , respectively, the proof is analogous to the one of Theorem 2.1.

\subsection{Theorem 3.4}

\subsubsection{Lemma used for proving Theorem 3.4}

Since $\left[D^{\circ}\left(\tilde{K}_{1}, Y\right), d_{1}^{*}\right]$ and $\left[D^{\circ}\left(\tilde{K}_{1}, Y\right), d_{1}^{\circ}\right]$ are separable, both $\left[\mathcal{D}^{\circ}\left(\tilde{K}_{1}, Y\right)\right.$, $\left.d_{1}^{*}\right]$ and $\left[\mathcal{D}^{\circ}\left(\tilde{K}_{1}, Y\right), d_{1}^{\circ}\right]$ are equivalent to the ball $\sigma$-fields generated by the open balls in the two metric spaces. In the following, the counterpart of Lemma 4.3 for the Borel $\sigma$-field $\left[\mathcal{D}^{\circ}\left(\tilde{K}_{1}, Y\right), d_{1}^{*}\right]$ or $\left[\mathcal{D}^{\circ}\left(\tilde{K}_{1}, Y\right), d_{1}^{\circ}\right]$ is given. Let $\tilde{K}_{1}^{*}$ be a dense set of $\tilde{K}_{1}$ and contain the equivalence class [1].

Lemma 5.6. $\sigma\left(\pi_{x}: x \in \tilde{K}_{1}^{*}\right)=\mathcal{D}^{\circ}\left(\tilde{K}_{1}, Y\right)$ and $\left\{\pi_{x_{1} \cdots x_{k}}^{-1}\left(S^{k}\right): S^{k} \in\right.$ $\left.\mathcal{Y}^{k}, x_{1}, \ldots, x_{k} \in \tilde{K}_{1}^{*}\right\}$ is a separating class of $\mathcal{D}^{\circ}\left(\tilde{K}_{1}, Y\right)$, where $\pi_{x_{1} \cdots x_{k}}$ : $D^{\circ}\left(\tilde{K}_{1}, Y\right) \rightarrow Y^{k}$ is the projection operator defined on $D^{\circ}\left(\tilde{K}_{1}, Y\right)$ and where $D^{\circ}\left(\tilde{K}_{1}, Y\right)$ and $\mathcal{D}^{\circ}\left(\tilde{K}_{1}, Y\right)$ are the space and the Borel $\sigma$-field, respectively, either in the sense of the metric $d_{1}^{*}$ or the metric $d_{1}^{\circ}$.

Proof. Since $\sigma\left(\pi_{x}: x \in \tilde{K}_{1}^{*}\right) \subset\left[\mathcal{D}^{\circ}\left(\tilde{K}_{1}, Y\right), d_{1}^{*}\right]$, it suffices to prove that $\left[\mathcal{D}^{\circ}\left(\tilde{K}_{1}, Y\right), d_{1}^{*}\right] \subset \sigma\left(\pi_{x}: x \in \tilde{K}_{1}^{*}\right)$.

Let $\hat{F}_{\Delta},\left\{x_{n i}\right\}$, and $\left[t_{n(i-1)}, t_{n i}\right)$, be the operator and sets given in the proof of Theorem 3.3. Let $\pi_{\tilde{K}_{\Delta}}$ be the projection random variable corresponding to the set $\tilde{K}_{\Delta}=\left(\left\{x_{n i}\right\} \cup\left\{x_{q 1}\right\}\right)$, where $\left(\left\{x_{n i}\right\} \cup\left\{x_{q 1}\right\}\right) \subset \tilde{K}_{1}^{*}$. Note that $\pi_{\tilde{K}_{\Delta}}$ is measurable with respect to $\sigma\left(\pi_{x}: x \in \tilde{K}_{1}^{*}\right)$ and $\mathcal{Y}^{N}$, where $N$ is the number of elements in $\tilde{K}_{\Delta}$. Let the random variable $\mathcal{V}_{\Delta}: Y^{N} \rightarrow$ $D^{\circ}\left(\tilde{K}_{1}, Y\right)$ take value $\mathcal{V}_{\Delta}(z) \in D^{\circ}\left(\tilde{K}_{1}, Y\right)$ and the operator $\mathcal{V}_{\Delta}(z)$ take constant values $z_{n i}$ over the set $\left[t_{n(i-1)}, t_{n i}\right)$ and $z_{q 1}$ over $B^{*}\left(x_{q 1}, \Delta\right)$, where $z_{n i}$ and $z_{q 1}$ are the elements of $z$, the number of elements in the set $\left\{z_{n i}\right\} \cup\left\{z_{q 1}\right\}$ is $N$, and $z \in Y^{N}$. Then, $\mathcal{V}_{\Delta}$ is continuous because

$$
d_{1}^{*}\left[\mathcal{V}_{\Delta}\left(z_{m}\right), \mathcal{V}_{\Delta}(z)\right] \leq\left\|\mathcal{V}_{\Delta}\left(z_{m}\right)-\mathcal{V}_{\Delta}(z)\right\|_{s u p}
$$


and $\left\|\mathcal{V}_{\Delta}\left(z_{m}\right)-\mathcal{V}_{\Delta}(z)\right\|_{\text {sup }} \underset{m \rightarrow \infty}{\longrightarrow} 0$ as $z_{m} \underset{m \rightarrow \infty}{\longrightarrow} z$. Thus, $\mathcal{V}_{\Delta} \pi_{\tilde{K}_{\Delta}}$ is measurable with respect to $\sigma\left(\pi_{x}: x \in \tilde{K}_{1}^{*}\right)$ and $\left[\mathcal{D}^{\circ}\left(\tilde{K}_{1}, Y\right), d_{1}^{*}\right]$ and $V_{\Delta} \pi_{\tilde{K}_{\Delta}}(F)=\hat{F}_{\Delta}$. Since

$$
\begin{aligned}
d_{1}^{*}\left[V_{\Delta} \pi_{\tilde{K}_{\Delta}}(F), F\right] & =d_{1}^{*}\left(\hat{F}_{\Delta}, F\right) \\
& \leq \max \left[w_{1}^{\prime \prime}(F, \Delta), \Delta\right]
\end{aligned}
$$

(see the proof of Theorem 3.2 given in the supplementary materials), there exists a sequence $\left\{\Delta_{n}\right\}$ such that for any $F \in D^{\circ}\left(\tilde{K}_{1}, Y\right), d_{1}^{*}\left[V_{\Delta_{n}} \pi_{\tilde{K}_{\Delta_{n}}}(F), F\right]$ $\underset{n \rightarrow \infty}{\longrightarrow} 0$ as $\Delta_{n} \underset{n \rightarrow \infty}{\longrightarrow} 0$. Further, because $D^{\circ}\left(\tilde{K}_{1}, Y\right)$ is separable, the identity operator $I(F)=F=\lim _{n \rightarrow \infty} V_{\Delta_{n}} \pi_{\tilde{K}_{\Delta_{n}}}(F)$ is measurable with respect to $\sigma\left(\pi_{x}: x \in \tilde{K}_{1}^{*}\right)$ and $\left[\mathcal{D}^{\circ}\left(\tilde{K}_{1}, Y\right), d_{1}^{*}\right]$ by Corollary 4.30 of [3]. Thus, $\left[\mathcal{D}^{\circ}\left(\tilde{K}_{1}, Y\right), d_{1}^{*}\right] \subset \sigma\left(\pi_{x}: x \in \tilde{K}_{1}^{*}\right)$. Moreover, $\left[\mathcal{D}^{\circ}\left(\tilde{K}_{1}, Y\right), d_{1}^{\circ}\right] \subset \sigma\left(\pi_{x}: x \in\right.$ $\tilde{K}_{1}^{*}$ ) because $d_{1}^{\circ}$ and $d_{1}^{*}$ are equivalent.

Since the class of finite-dimensional sets $\left\{\pi_{x_{1} \cdots x_{k}}^{-1}\left(S^{k}\right): S^{k} \in \mathcal{Y}^{k}, x_{1}, \ldots\right.$, $\left.x_{k} \in \tilde{K}_{1}^{*}\right\}$ is a $\pi$-system and generates $\sigma\left(\pi_{x}: x \in \tilde{K}_{1}^{*}\right)$, it is a separating class for $\mathcal{D}^{\circ}\left(\tilde{K}_{1}, Y\right)$.

\subsubsection{Proof of Theorem 3.4}

For any probability measure $Q$, let $\tilde{K}_{1 Q}^{*}$ satisfy that $\pi_{x}$ on $\left[D^{\circ}\left(\tilde{K}_{1}, Y\right), d_{1}^{*}\right]$ or $\left[D^{\circ}\left(\tilde{K}_{1}, Y\right), d_{1}^{\circ}\right]$ is continuous except the set of points of $Q$-measure 0 for each $x \in \tilde{K}_{1 Q}^{*}$. Then, $\tilde{K}_{1 Q}^{*}$ is the dense set of $\tilde{K}_{1}$, contains the equivalence class [1], and $\left(\tilde{K}_{1 Q}^{*}\right)^{c} \cap \tilde{K}_{1}$ contains only countable equivalence classes $[c]$. The properties of the set are proved as follows. Let $J_{[c]}=\left\{F: F\left(x^{-}\right) \neq F(x), x \in\right.$ $[c]\}$ and $J_{[c], 1 / n}=\left\{F:\left\|F(x)-F\left(x^{-}\right)\right\|_{Y}>1 / n, x \in[c]\right\}$, where $0<c<1$. For every fixed positive integers $m$ and $n$, if there are infinitely many sets $J_{\left[x_{m n i}\right], 1 / n}$ for which $Q\left(J_{\left[x_{m n i}\right], 1 / n}\right) \geq 1 / m$, then $Q\left(\lim \sup _{i \rightarrow \infty} J_{\left[x_{m n i}\right], 1 / n}\right) \geq$ $1 / m$ which contradicts with the fact that at most finitely many equivalence classes $\left[c_{n i}\right]$ satisfy $\sup _{x \in\left[c_{n i}\right]}\left\|F\left(x^{-}\right)-F(x)\right\|_{Y} \geq 1 / n$ by the equation $\lim _{\Delta \rightarrow 0} w_{1}^{\prime}(F, \Delta)=0$, i.e., only finitely many equivalence classes with "jumps" exceeding or equal to $1 / n$. Therefore, there can be only finitely many equivalence classes $\left[x_{m n i}\right]$ satisfying $Q\left(J_{\left[x_{m n i}\right], 1 / n}\right) \geq 1 / m$. Let $\tilde{K}_{1 Q}=$ $\tilde{K}_{1} \cap\left(\cup_{m} \cup_{n} \cup_{i}\left[x_{m n i}\right]\right)^{c}$ and hence $Q\left(J_{[c]}\right)=\lim _{n \rightarrow \infty} Q\left(J_{[c], 1 / n}\right)=0$ for 
$c \in \tilde{K}_{1 Q}$ owing to $J_{[c], 1 / n} \uparrow J_{[c]}$, where $J_{[c], 1 / n} \uparrow J_{[c]}$ is denoted as $J_{[c], 1} \subset$ $J_{[c], 1 / 2} \subset \cdots, \pi_{x}$ is continuous as $x \in[1]$ owing to

$$
\left\|\pi_{x}\left(F_{n}\right)-\pi_{x}(F)\right\|_{Y}=\left\|F_{n}(x)-F(x)\right\|_{Y} \leq d_{1}^{*}\left(F_{n}, F\right) \leq d_{1}^{\circ}\left(F_{n}, F\right),
$$

for $x \in[1]$ and $\tilde{K}_{1 Q} \subset \tilde{K}_{1 Q}^{*}, \tilde{K}_{1 Q}^{*}$ has the required properties.

Next, by the tightness of $\left\{P_{n}\right\}$, there exists a further subsequence $\left\{P_{n_{i(m)}}\right\}$ of every subsequence $\left\{P_{n_{i}}\right\}$ of $\left\{P_{n}\right\}$ converging weakly to a probability measure $Q$, i.e., $P_{n_{i(m)}} \Rightarrow Q$. Then, $P_{n_{i(m)}} \pi_{x_{1} \ldots x_{k}}^{-1} \Rightarrow Q \pi_{x_{1} \ldots x_{k}}^{-1}$ for all $x_{1}, \ldots, x_{k} \in \tilde{K}_{1 Q}^{*}$ by the mapping theorem. Thus, let $\tilde{K}_{1}^{* *}=\tilde{K}_{1 P}^{*} \cap \tilde{K}_{1 Q}^{*} \cdot \tilde{K}_{1}^{* *}$ is a dense set of $\tilde{K}_{1}$ and contains the equivalence class [1] because $\left(\tilde{K}_{1}^{* *}\right)^{c} \cap \tilde{K}_{1}$ contains only countable equivalence classes and both $\tilde{K}_{1 P}^{*}$ and $\tilde{K}_{1 Q}^{*}$ contain the equivalence class [1]. Then, by weak convergence of the finite dimensional distributions of $\left\{P_{n}\right\}$ to $P$ in $\tilde{K}_{1}^{* *}, P=Q$ because $P \pi_{x_{1} \ldots x_{k}}^{-1}=Q \pi_{x_{1} \ldots x_{k}}^{-1}$ for $x_{1}, \ldots, x_{k} \in \tilde{K}_{1}^{* *}$ and the class of finite-dimensional sets $\left\{\pi_{x_{1} \cdots x_{k}}^{-1}\left(S^{k}\right): S^{k} \in\right.$ $\left.\mathcal{Y}^{k}, x_{1}, \ldots, x_{k} \in \tilde{K}_{1}^{* *}\right\}$ is a separating class by Lemma 5.6. Finally, by Theorem 2.6 of [4], the result follows.

\section{References}

1. Y. A. Abramovich and C. D. Aliprantis, An Invitation to Operator Theory, American Mathematical Society, Rhode Island, 2002.

2. N. I. Akhiezer and I. M. Glazman, Theory of Linear Operators in Hilbert Space, Dover Publications, New York, 1993.

3. C. D. Aliprantis and K. C. Border, Infinite Dimensional Analysis: A Hitchhiker's Guide, 2nd ed., Springer-Verlag, Berlin Heidelberg, 1999.

4. P. Billingsley, Convergence of Probability Measures, 2nd ed., Wiley, New York, 1999.

5. R. M. Dudley, Uniform Central Limit Theorems, Cambridge University Press, Cambridge, 1999.

6. R. M. Dudley, Real Analysis and Probability, Cambridge University Press, Cambridge, 2002.

7. R. Durrett, Stochastic Calculus: A Practical Introduction, CRC Press, Boca Raton, 1996.

8. L. C. Evans, Partial Differential Equations, American Mathematical Society, Rhode Island, 1998.

9. N. V. Krylov, Introduction to the Theory of Random Processes, American Mathematical Society, Rhode Island, 2002. 
10. M. Ledoux and M. Talagrand, Probability in Banach Spaces, Springer-Verlag, Berlin Heidelberg, 1991.

11. T. Lindvall, Weak convergence in the function space $D[0, \infty), J$. Appl. Probab., 10 (1973), 109-121.

12. J. R. Munkres, Topology, 2nd ed., Prentice Hall, Upper Saddle River, 2000.

13. J. W. Neuberger, Review: Pilz, G. Near-rings, the theory and its application, Bull. Amer. Math. Soc., 84 (1978), 934-937.

14. W. H. Wei, Robust estimation: Location-scale and regression problems, Taiwanese J. Math., 117 (2013), 1055-1093.

15. W. H. Wei, On finite dimensional approximation in nonparametric regression, J. Chinese Statist. Assoc., 2016.

16. E. Zeidler, Applied Functional Analysis: Application to Mathematical Physics, Springer-Verlag, New York, 1995. 